\title{
ASASSN-15pz: Revealing Significant Photometric Diversity among 2009dc-like, Peculiar SNe Ia*
}

Ping Chen ${ }^{1,2}$ (D) , Subo Dong $^{1}$ (iD), Boaz Katz ${ }^{3}$ (D) C. S. Kochanek ${ }^{4,5}$ (iD) Juna A. Kollmeier ${ }^{6}$ (D), K. Maguire ${ }^{7,8}$, M. M. Phillips ${ }^{9}$ (iD), J. L. Prieto ${ }^{10,11}$ (D) B. J. Shappee ${ }^{12}$ (D), M. D. Stritzinger ${ }^{13}$ (D) Subhash Bose $^{1}$ (D), Peter J. Brown ${ }^{14,15}$ (D), T. W.-S. Holoien ${ }^{16}$ (D), L. Galbany ${ }^{17}$ (D), Peter A. Milne ${ }^{18}$ (ID) Nidia Morrell $^{9}$ (D), Anthony L. Piro ${ }^{19}$ (D), K. Z. Stanek ${ }^{4,5}$, Todd A. Thompson ${ }^{4,5,20}$, and D. R. Young 21 (iD

${ }^{1}$ Kavli Institute for Astronomy and Astrophysics, Peking University, Yi He Yuan Road 5, Hai Dian District, Beijing 100871, People's Republic of China dongsubo@pku.edu.cn

${ }^{2}$ Department of Astronomy, School of Physics, Peking University, Yi He Yuan Road 5, Hai Dian District, Beijing 100871, People's Republic of China ${ }^{3}$ Department of Particle Physics and Astrophysics, Weizmann Institute of Science, Rehovot 76100, Israel

${ }^{4}$ Department of Astronomy, The Ohio State University, 140 West 18th Avenue, Columbus, OH 43210, USA

${ }^{5}$ Center for Cosmology and AstroParticle Physics, The Ohio State University, 191 W. Woodruff Ave., Columbus, OH 43210, USA

${ }^{6}$ Observatories of the Carnegie Institution for Science, 813 Santa Barbara Street, Pasadena, CA 91101, USA

7 Astrophysics Research Centre, School of Mathematics and Physics, Queen's University Belfast, Belfast BT7 1NN, UK

${ }^{8}$ School of Physics, Trinity College Dublin, Dublin 2, Ireland

${ }^{9}$ Las Campanas Observatory, Carnegie Observatories, Casilla 601, La Serena, Chile

${ }^{10}$ Núcleo de Astronomía, Facultad de Ingeniería y Ciencias, Universidad Diego Portales, Ejército 441, Santiago, Chile ${ }^{11}$ Millennium Institute of Astrophysics, Santiago, Chile

${ }^{12}$ Institute for Astronomy, University of Hawaii, 2680 Woodlawn Drive, Honolulu, HI 96822, USA

13 Department of Physics and Astronomy, Aarhus University, Ny Munkegade 120, DK-8000 Aarhus C, Denmark

${ }^{14}$ Department of Physics and Astronomy, Texas A\&M University, 4242 TAMU, College Station, TX 77843, USA

${ }^{15}$ George P. and Cynthia Woods Mitchell Institute for Fundamental Physics \& Astronomy, USA

${ }^{16}$ The Observatories of the Carnegie Institution for Science, 813 Santa Barbara Street, Pasadena, CA 91101, USA

${ }^{17}$ PITT PACC, Department of Physics and Astronomy, University of Pittsburgh, Pittsburgh, PA 15260, USA

${ }^{18}$ University of Arizona, Steward Observatory, 933 North Cherry Avenue, Tucson, AZ 85719, USA

${ }^{19}$ Observatories of the Carnegie Institution for Science, 813 Santa Barbara Street, Pasadena, CA 91101, USA

${ }^{20}$ Institute for Advanced Study, 1 Einstein Drive, Princeton, NJ 08540, USA

${ }^{21}$ Astrophysics Research Centre, School of Mathematics and Physics, Queens University Belfast, Belfast BT7 1NN, UK Received 2019 April 5; revised 2019 May 16; accepted 2019 May 31; published 2019 July 22

\begin{abstract}
We report comprehensive multi-wavelength observations of a peculiar Type Ia-like supernova ("SN Ia-pec") ASASSN-15pz. ASASSN-15pz is a spectroscopic "twin" of SN 2009dc, a so-called "Super-Chandrasekhar-mass" $\mathrm{SN}$, throughout its evolution, but it has a peak luminosity $M_{B \text {,peak }}=-19.69 \pm 0.12$ mag that is $\approx 0.6$ mag dimmer and comparable to the SN $1991 \mathrm{~T}$ sub-class of SNe Ia at the luminous end of the normal width-luminosity relation. The synthesized ${ }^{56} \mathrm{Ni}$ mass of ${ }^{{ }^{56} \mathrm{Ni}}=1.13 \pm 0.14 M_{\odot}$ is also substantially less than that found for several 2009dc-like SNe. Previous well-studied 2009dc-like SNe have generally suffered from large and uncertain amounts of host-galaxy extinction, which is negligible for ASASSN-15pz. Based on the color of ASASSN-15pz, we estimate a host extinction for SN 2009dc of $E(B-V)_{\text {host }}=0.12$ mag and confirm its high luminosity $\left(M_{B \text {, peak }}[2009 \mathrm{dc}] \approx-20.3 \mathrm{mag}\right)$. The $2009 \mathrm{dc}-$ like $\mathrm{SN}$ population, which represents $\sim 1 \%$ of SNe Ia, exhibits a range of peak luminosities, and do not fit onto the tight width-luminosity relation. Their optical light curves also show significant diversity of late-time ( $~ 50$ days) decline rates. The nebular-phase spectra provide powerful diagnostics to identify the 2009 dc-like events as a distinct class of SNe Ia. We suggest referring to these sources using the phenomenology-based "2009dc-like SN Ia-pec" instead of "Super-Chandrasekhar SN Ia," which is based on an uncertain theoretical interpretation.
\end{abstract}

Key words: supernovae: general - supernovae: individual (ASASSN-15pz)

\section{Introduction}

The empirical correlations between peak luminosity and lightcurve width (or the post-peak decline rate; see the yellow filled circles in the left panel of Figure 1) established by Phillips (1993) make Type Ia supernovae (SNe Ia) important standardizable candles for cosmology. Yet, the progenitor systems and explosion mechanism of SNe Ia are still under intense debate (e.g., see the reviews by Maoz et al. 2014; Wang 2018). The width-luminosity relation (Pskovskii 1977; Phillips 1993, 2005) may also hold important clues to understanding the physics of the SNe Ia population (e.g., Wygoda et al. 2019a, 2019b), and these

\footnotetext{
* This paper includes data gathered with the $6.5 \mathrm{~m}$ Magellan Telescopes located at Las Campanas Observatory, Chile.
}

relations appear to connect the dimmest 1991bg-like events (Filippenko et al. 1992a; Leibundgut et al. 1993; Turatto et al. 1996), the most luminous 1991T-like events (Filippenko et al. 1992b; Jeffery et al. 1992; Ruiz-Lapuente et al. 1992), and the "normal" SNe Ia used for cosmology into a continuous distribution (e.g., see Figure 4 of Burns et al. 2018 and the yellow filled circles in the right panel of Figure 1). Evidence for the continuity of the SN Ia population includes other light-curve properties (e.g., Phillips 2012; Burns et al. 2014), early-phase spectroscopic properties (e.g., Nugent et al. 1995; Branch et al. 2009), and nebular-phase spectroscopic properties (e.g., Mazzali et al. 1998; Kushnir et al. 2013; Dong et al. 2018).

In recent years, wide-field sky surveys have discovered an increasing number of peculiar and luminous SNe Ia-like 


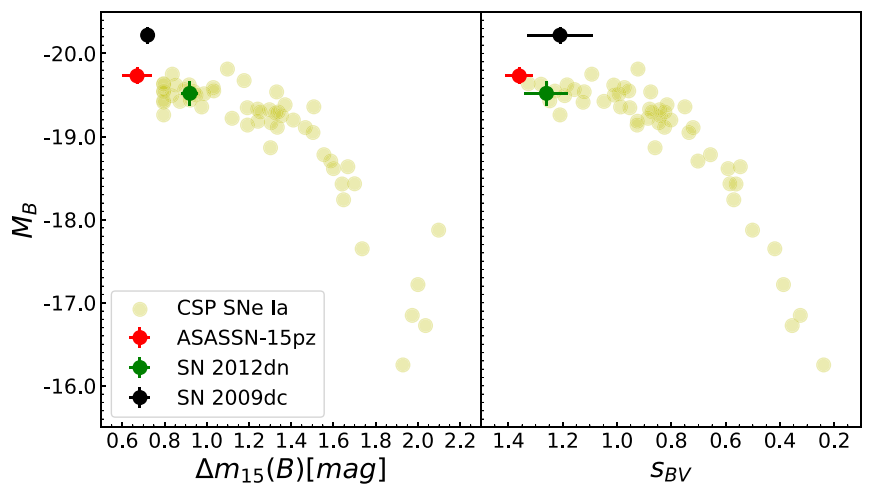

Figure 1. Width vs. luminosity (decline rate) relation (Phillips 1993) populated with a subset of CSP-I SNe Ia from Burns et al. (2014). The relation is parameterized by $\Delta m_{15}$ (left) and $s_{B V}$ (right). The absolute $B$-band magnitudes are extinction-corrected as described by Burns et al. 2014, and distances are computed using a Hubble constant of $H_{0}=73 \mathrm{~km} \mathrm{~s}^{-1} \mathrm{Mpc}^{-1}$ for SNe Ia with redshift $z>0.01$ and redshift-independent distances from the literature for objects with $z<0.01$.

events. These objects are spectroscopically similar to $\mathrm{SNe}$ Ia, especially the 1991T-like sub-class, near maximum light, but they often exhibit spectroscopic peculiarities and also usually deviate from the Phillips relation (see, e.g., Taubenberger 2017). In particular, there are SNe Ia-like objects with peak absolute magnitudes of $M_{V}^{\text {peak }} \simeq-20$ that are greater than those of the most luminous SNe Ia (i.e., the 1991T-like subclass) on the Phillips relation, leading to speculation that their progenitor masses may be super-Chandrasekhar. Howell et al. (2006) found the first such event, SN 2003fg, and a small number of similarly luminous SNe Ia-like events (referred to in the following text as luminous peculiar SNe Ia or "luminous SNe Ia-pec" in short) have been discovered later: SN 2006gz (Hicken et al. 2007), SN 2007if (Scalzo et al. 2010) and SN 2009dc (Yamanaka et al. 2009a; Silverman et al. 2011; Taubenberger et al. 2011). Their spectra have substantial differences from SNe Ia on the Phillips relation. In general, they appear to be hotter and show evidence of unburned carbon in their early-phase spectra and weak or even absent [Fe III] emission in their nebular spectra. The luminosity estimates for these objects, including SN 2009dc, have suffered from uncertainties in the host-galaxy extinction. In the case of $\mathrm{SNe}$ Ia on the Phillips relation, there is a remarkable uniformity to the intrinsic $(B-V)$ color between 30 and 90 days (i.e., the Lira relation; Phillips et al. 1999) for objects with negligible host extinction, allowing accurate estimates for host-reddened $\mathrm{SNe}$ Ia. However, the luminous SNe Ia-pec found to date all suffer from significant host-reddening and their intrinsic colors remain unclear.

In particular, the host extinction of the best studied example, SN 2009dc, is uncertain. Its existence is clearly due to the presence of a NaiD absorption line with an equivalent width of EW(Na I D) $\approx 1.0 \AA$ (Silverman et al. 2011; Taubenberger et al. 2011). Taubenberger et al. (2011) adopted an extinction of $E(B-V)_{\text {host }}=0.10 \pm 0.07 \mathrm{mag}$. They noted that the postpeak $(B-V)$ curve of SN 2009dc does not follow the Lira relation and is redder by $\sim 0.3 \mathrm{mag}$ at 90 days. Considering various empirical relations between $\mathrm{EW}(\mathrm{NaID})$ and dust extinction, Silverman et al. (2011) found a range of $0.1<$ $E(B-V)_{\text {host }}<0.3$ mag that translates into a significant uncertainty of $\sim 0.6 \mathrm{mag}$ in the peak luminosity. Another luminous SN Ia-pec, SN 2006gz, also suffers from the uncertainties in its host-galaxy extinction. If the Lira relation is adopted, the host extinction is $E(B-V)_{\text {host }}=0.18$ mag, implying a peak absolute magnitude of $M_{V}^{\text {peak }}=-19.74 \pm 0.16$ (Hicken et al. 2007). SN 2012dn was found to be a spectroscopic "clone" of SN 2006gz (Chakradhari et al. 2014), while its estimated peak luminosities of $M_{B}^{\text {peak }}=-19.52 \pm 0.15 \mathrm{mag}$ and $M_{V}^{\text {peak }}=$ $-19.42 \pm 0.15 \mathrm{mag}$ were similar to an average $\mathrm{SN}$ Ia but substantially dimmer than previously known 2009dc-like SNe. SN 2012dn was also subject to large uncertainty in host extinction estimates.

Here we report the discovery and observations of ASASSN$15 \mathrm{pz}$, which is nearly identical to SN 2009dc spectroscopically, but has some significantly different photometric properties including its peak luminosity and late-time light-curve decline rates. Our UV, optical, and near-infrared (NIR) photometry data and visual-wavelength spectra of ASASSN-15pz are described in Section 2. In Section 3, we show that ASASSN$15 \mathrm{pz}$ is essentially free from host-galaxy extinction, allowing the luminosity and intrinsic color evolution of ASASSN-15pz to be accurately determined and compared to other SNe. Our spectroscopic data span from before the peak into the nebular phase, and we show in Section 4 that ASASSN-15pz is a spectroscopic "twin" of SN 2009dc (see Section 4). We discuss the implications of our work for understanding this class of SNe Ia in Section 5.

\section{Observations and Data Reduction}

ASASSN-15pz $\quad$ (R.A. $=03^{\mathrm{h}} 08^{\mathrm{m}} 48.443, \quad$ decl. $=$ $-35^{\mathrm{d}} 13^{\mathrm{m}} 50$. 89 ) was discovered by the All-sky Automated Survey for SuperNovae (ASAS-SN; Shappee et al. 2014) on UT 2015 September 27.16 (JD = 2457292.66) at an apparent $V$-band magnitude of $\sim 16.4 \mathrm{mag}$ (Brimacombe et al. 2015). The source is approximately 16". 34 North and 14!" 45 West of the nearby SB(rs)d galaxy ESO 357-G005 (6dF J0308495351409) at $z=0.014837$ (Jones et al. 2009). An image of ASASSN-15pz is shown in Figure 2, and its projected distance is $6.5 \mathrm{kpc}$ from the center of the host galaxy. Correcting for the infall velocity of the Local Group toward the Virgo Cluster leads to a luminosity distance of $d_{L}=58.8 \pm 3.3 \mathrm{Mpc}$ (distance modulus $\mu=33.85 \pm 0.12 \mathrm{mag}$ ) for $H_{0}=73 \mathrm{~km} \mathrm{~s}^{-1} \mathrm{Mpc}^{-1}$ (Burns et al. 2018), $\Omega_{m}=0.27$, and $\Omega_{\Lambda}=0.73$. For consistency, the distance moduli for all objects in Table 3 are computed in the same way as for ASASSN-15pz. We include an uncertainty of $\sigma_{\mathrm{cz}}=250 \mathrm{~km} \mathrm{~s}^{-1}$ (see, e.g., Burns et al. 2018) to account for peculiar velocities.

ASASSN-15pz was classified as a SN Ia by an optical spectrum taken with Nordic Optical Telescope (NOT) on UT 2015 September 30.16 (Taddia \& Stritzinger 2015). We performed optical follow-up imaging observations starting from its discovery as part of systematic efforts to follow up a large, complete and volume-limited $(z<0.02)$ complete sample of SNe Ia (P. Chen et al. 2019, in preparation). It peaked in $B$ band at $14.2 \mathrm{mag}$ on UT 2015 October 11.72 (JD 2457307.22), which is used as the phase reference epoch throughout the text. The presence of a prominent $\mathrm{C}$ II absorption feature redward of the Si II $\lambda 6355$ feature, similar to the luminous SNe Ia-pec SN 2006gz and SN 2009dc, motivated us to follow this event extensively. We initiated Neil Gehrels Swift Observatory (Swift; Gehrels et al. 2004) Ultraviolet Optical Telescope (UVOT) observation around its peak and NIR photometric observations around 10 days after $B$-band maximum. A total of eight optical spectra were 


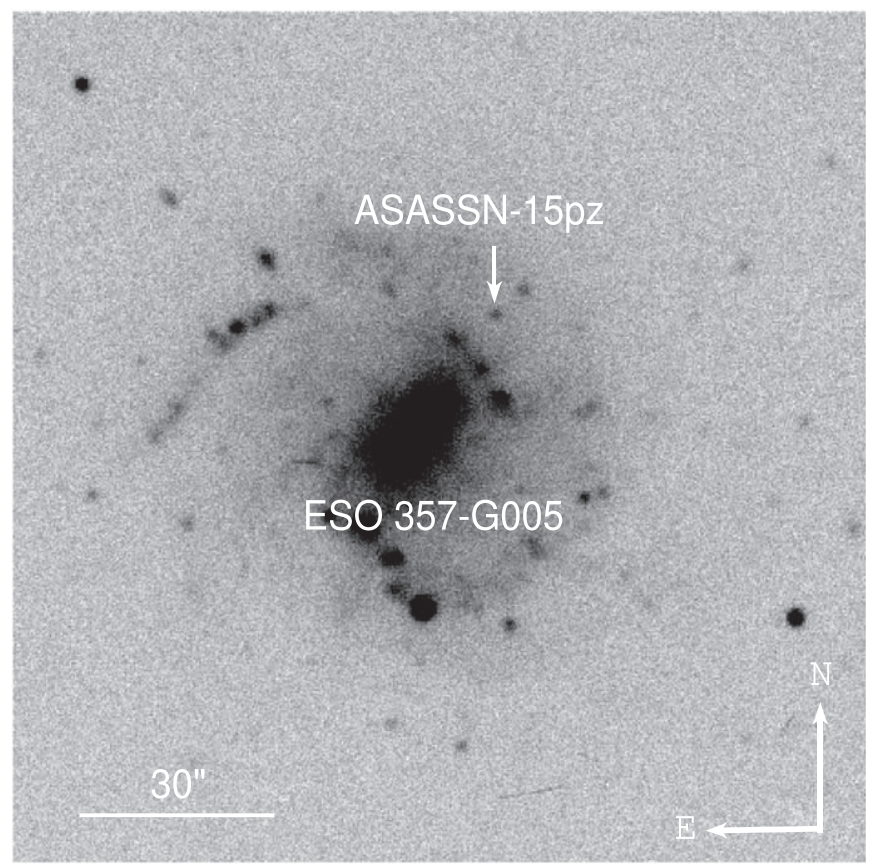

Figure 2. SDSS $r$-band image of ASASSN-15pz in the host-galaxy ESO 357 G005. The image was taken with Clay/LDSS3C on UT 2016 June 7.40 $(\mathrm{JD}=2457546.90)$.

obtained for ASASSN-15pz during the follow-up campaign, spanning from before the peak and into the nebular phase. We summarize our photometric and spectroscopic observations in Appendices $\mathrm{A}$ and $\mathrm{B}$, with the photometric results reported in Tables 4-6 and the spectroscopic observing logs given in Table 7.

\section{Photometric Evolution of ASASSN-15pz}

The UV, optical, and NIR light curves of ASASSN-15pz are shown in Figure 3. Our extensive photometric coverage makes it one of the best observed luminous SNe Ia-pec.

\subsection{Optical and NIR Light Curves}

A polynomial fit to the $B$-band light curve around maximum brightness yields $B_{\max }=14.23 \pm 0.02 \mathrm{mag}$ on JD $2457307.2 \pm 0.8$ (UT 2015 Oct 11.7). Our multi-band optical photometry observations span the phases from -13 days to +163.0 days. The $V$-band light curve peaked at $14.23 \pm$ $0.01 \mathrm{mag}$ on JD $2457307.2 \pm 0.7$. We derive an observed $\Delta m_{15}(B)=0.67 \pm 0.07 \mathrm{mag}$, which makes ASASSN-15pz one of the slowest-declining SNe Ia known. The decline rate is similar to other luminous SNe Ia-pec such as SN 2006gz $\left(\Delta m_{15}(B)=0.69 \pm 0.04 \mathrm{mag}\right.$; Hicken et al. 2007), and SN 2009dc $\left(\Delta m_{15}(B)=0.72 \pm 0.03 \quad\right.$ mag; Taubenberger et al. 2011), while it is slower than SN 2012dn (0.92 \pm 0.04 mag; Chakradhari et al. 2014). Other key photometric parameters derived from the optical light curves of ASASSN$15 \mathrm{pz}$ are tabulated in Table 1.

In Figure 4 , we compare the $B$ - and $V$-band light curves of ASASSN-15pz (red filled circles) to those of SN 2009dc (black filled circles), SN 2012dn (green filled circles), and the "normal" SNe Ia (yellow lines) from the Carnegie Supernova Project-I (CSP-I; Burns et al. 2014). In the left two panels, the absolute-magnitude light curves are corrected for both the Milky Way (MW) and host-galaxy extinctions. For

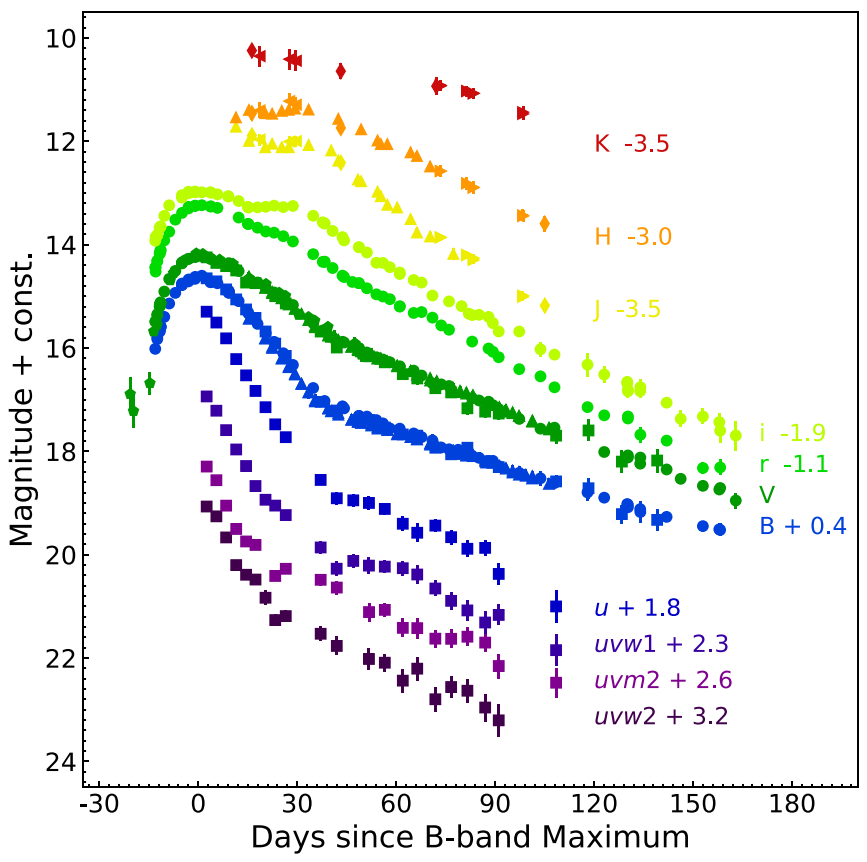

Figure 3. Multi-band light curves of ASASSN-15pz. This includes data obtained with Swift (squares), LCOGT (circles), SMARTS (upward-facing triangles), NOT (diamonds), and UKIRT (rightward-facing triangles for WFCAM and left-facing triangles for UFTI).

SN 2009dc, SN 2012dn, and ASASSN-15pz, the adopted reddening parameters and distance modulus are listed in Table 3, and we assume $R_{V}=3.1$. We discuss how the reddening parameters are derived in Section 3.3. For the CSP-I sample, we used the extinction corrections from Burns et al. (2014). ASASSN-15pz is comparable to the most luminous SNe Ia in the CSP-I sample, while it is significantly dimmer than SN 2009dc. SN 2012dn is $\sim 0.2 \mathrm{mag}$ dimmer than ASASSN-15pz and is consistent with the typical 1991T/ 1999aa sub-class of SNe Ia. In the right two panels, the $B$ - and $V$-band light curves are normalized to the fluxes at peak. The insets highlight the light-curve shapes near maximum, and ASASSN-15pz and SN 2009dc have the broadest light curves. They also stand out in the left panel of Figure 1 when using $\Delta m 15(B)$ to measure post-peak light-curve decline rate. ASASSN-15pz and SN 2009dc have nearly identical $B$ - and $V$-band light-curve shapes in the early phases $(\lesssim 50$ days). Between 50 and 150 days, ASASSN-15pz declines in the $B$ band ( $V$ band) at a rate of $0.023 \mathrm{mag} \mathrm{day}^{-1}\left(0.027 \mathrm{mag} \mathrm{day}^{-1}\right)$, which is substantially faster than SN $2009 \mathrm{dc}\left(0.015 \mathrm{mag}^{-1}{ }^{-1}\right.$ in $B$ and 0.019 mag day $^{-1}$ in $V$; Silverman et al. 2011). The late-time decline rate of ASASSN-15pz is comparable to the fastest declining $\mathrm{SNe}$ in the CSP-I sample. At late times, SN 2012dn declines even faster than ASASSN-15pz and all SNe Ia in the CSP-I sample. Chakradhari et al. (2014) argued that the fast late-time decline of SN 2012dn is due to dust formation.

Burns et al. (2014) analyzed the intrinsic colors of SNe Ia using the CSP-I sample and introduced a new light-curve parameter- "color stretch" $s_{B V}=\left(t_{B-V}^{\max } / 30\right.$ days $)$ where $t_{B-V}^{\max }$ is the time of $(B-V)$ color maximum after $B$-band maximum. They found a tight correlation between $s_{B V}$ and peak luminosity (see the right panel of Figure 1) for SNe Ia. This correlation between $s_{B V}$ and peak luminosity has significantly less scatter than that between $\Delta m_{15}(B)$ and peak luminosity for the lowluminosity $\mathrm{SNe}$ Ia $\left(M_{B} \gtrsim-19\right)$. If we fit the $(B-V)$ color 
Table 1

Photometric Parameters of ASASSN-15pz

\begin{tabular}{lcccccr}
\hline \hline Band & JD $^{\text {peak }}$ & $m_{\lambda}^{\text {peak }}$ & $M_{\lambda}^{\text {peak }}$ & $\Delta m_{15}(\lambda)$ & Decline rate $^{\text {a }}$ & ${\text { Color at } B_{\text {max }}}$ \\
\hline$B$ & $2457307.22 \pm 0.77$ & $14.23 \pm 0.02$ & $-19.69 \pm 0.12$ & $0.67 \pm 0.08$ & 2.265 & \\
$V$ & $2457307.15 \pm 0.71$ & $14.23 \pm 0.01$ & $-19.67 \pm 0.12$ & $0.38 \pm 0.04$ & 2.712 & $(B-V)_{0}=0.00 \pm 0.02$ \\
$r$ & $2457308.25 \pm 1.25$ & $14.36 \pm 0.02$ & $-19.53 \pm 0.12$ & $0.39 \pm 0.05$ & 3.277 & $(V-r)_{0}=-0.13 \pm 0.03$ \\
$i$ & $2457307.65 \pm 1.88$ & $14.86 \pm 0.02$ & $-19.02 \pm 0.12$ & $0.28 \pm 0.05$ & 3.432 & $(r-i)_{0}=-0.50 \pm 0.03$ \\
\hline
\end{tabular}

Note.

${ }^{a}$ Decline rate during 50-100 days after $B$-band maximum, in units of mag (100 days $)^{-1}$.

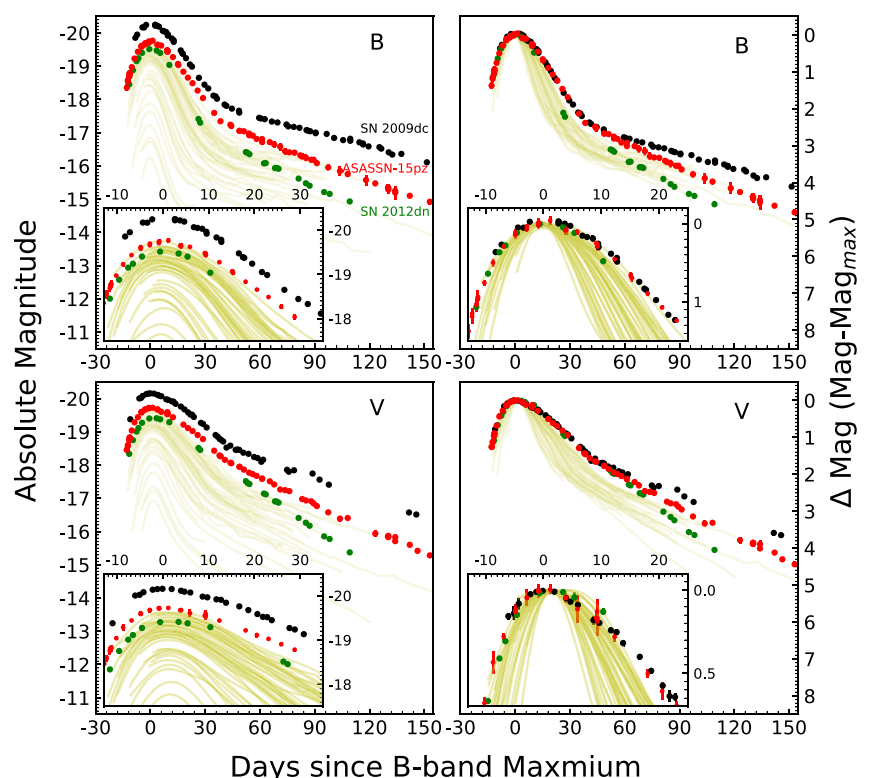

Figure 4. $B$ - (top) and $V$-band (bottom) light curves of ASASSN-15pz (red) compared to those of SN 2009dc (black), SN 2012dn (green) and SNe Ia from CSP-I (yellow). The left two panels show absolute magnitude light curves after correcting for Galactic and host-galaxy extinctions, while in the right two panels, all the light curves have been shifted to match at their peak magnitudes and to the time of $B$-band maximum.

evolution of ASASSN-15pz following the procedure of Burns et al. (2014), we obtain $t_{B-V}^{\max }=40.8 \pm 1.5$ days and $s_{B V}=1.36 \pm 0.05$.

We also apply the same fitting procedure to SN 2009dc, SN 2012dn, and the 1991T-like SN Ia SN 2005M with the best-fit models shown in Figure 5 and the parameters are given in Table 2. For comparison, the Lira relation from Burns et al. (2014) and its standard deviation are also shown. The latephase decline slope of ASASSN-15pz is similar to that of SN 2009dc, while slower than that of SN 2005M, which has a similar peak luminosity as ASASSN-15pz, and the Lira relation in general. We show in Section 3.3 that the host-galaxy extinction of ASASSN-15pz is negligible, and the measured $(B-V)$ color for ASASSN-15pz is thus intrinsic and can be used as an unreddened template for this sub-class.

In Figure 6 , the $r, i-, J$, and $H$-band light curves of ASASSN-15pz are compared with those of SN 2009dc, SN 2012dn and the CSP-I SNe Ia with good NIR light curves. Except for some low-luminosity SNe Ia, the $i J H$-band light curves of SNe Ia (including the 1991T-like sub-class) generally exhibit prominent secondary maxima. ASASSN-15pz, SN 2009dc and SN 2012dn all have secondary maxima, but unlike the SNe Ia from the CSP-I sample, they do not have significant troughs between the two peaks. Instead, their light

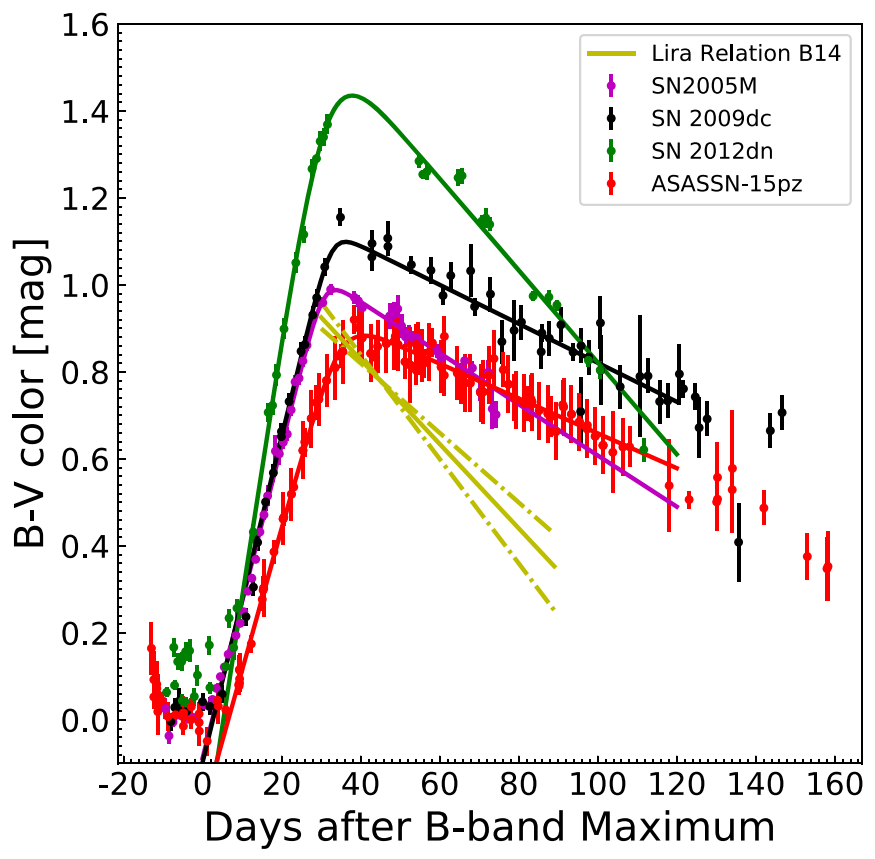

Figure 5. The $(B-V)$ color of ASASSN-15pz (red) compared with other overly luminous SNe Ia (SN 2009dc in black and SN 2012dn in green) and the 1991T-like SN 2005M in magenta. The mean and standard deviations of Lira relation from Burns et al. (2014) are shown as the yellow solid and dashed lines.

curves are "shoulder-like" between the two peaks with flat slopes. In the $H$-band, the peaks at $\gtrsim 30$ days after the $B$-band maximum are broad and luminous, and they appear to overwhelm the peaks near the $B$-band peaks.

\subsection{Swift UVOT Light Curve}

In Figure 7, we compare the evolution of ASASSN-15pz in $u, u v m 2,(u-v)$ and $(u v m 2-u)$ with SN 2009dc, SN 2012dn, and a few SNe Ia with good UV coverage, including the normal SN 2007af (Brown et al. 2009) and SN 2011by (Milne et al. 2013), the 1991bg-like SN 2005ke (Brown et al. 2009), and the "transitional" object SN 2007on (Gall et al. 2018), whose luminosity is between "normal" and 1991bg-like SNe Ia. It is well known that 2009 dc-like SNe are exceptionally UV bright around peak (see, e.g., Brown et al. 2014b), and ASASSN$15 \mathrm{pz}$ is also UV luminous. As shown in the bottom left panel of Figure 7, the $(u-v)$ colors of $\mathrm{SNe}$ Ia become redder after $B$-band maximum, then reverse and become bluer. Like the $(B-V)$ color curves, the $(u-v)$ color curves peak later for more luminous SNe. The (uvm2 $-u)$ color evolution of SNe Ia show more diversity, and ASASSN-15pz is the only object with $u v m 2$ detections at late phases ( $\gtrsim 50$ days) to show a flat, late-time $(u v m 2-u)$ color evolution. ASASSN-15pz, 
Table 2

Fitting Parameters of the $(B-V)$ Color Curve

\begin{tabular}{|c|c|c|c|c|c|}
\hline SN Name & $m_{15}(B)$ & $s_{0}$ & $s_{1}$ & $t_{B-V}^{\max }($ day $)$ & $s_{B V}$ \\
\hline $2005 \mathrm{M}$ & $0.87 \pm 0.02$ & $0.036 \pm 0.001$ & $-0.0059 \pm 0.0003$ & $33.6 \pm 0.8$ & $1.12 \pm 0.03$ \\
\hline $2009 \mathrm{dc}$ & $0.72 \pm 0.03$ & $0.037 \pm 0.003$ & $-0.0045 \pm 0.0005$ & $36.2 \pm 3.7$ & $1.21 \pm 0.12$ \\
\hline $2012 \mathrm{dn}$ & $0.92 \pm 0.04$ & $0.059 \pm 0.007$ & $-0.0106 \pm 0.0005$ & $37.9 \pm 2.5$ & $1.26 \pm 0.08$ \\
\hline ASASSN-15pz & $0.67 \pm 0.07$ & $0.033 \pm 0.003$ & $-0.0040 \pm 0.0002$ & $40.8 \pm 1.5$ & $1.36 \pm 0.05$ \\
\hline
\end{tabular}

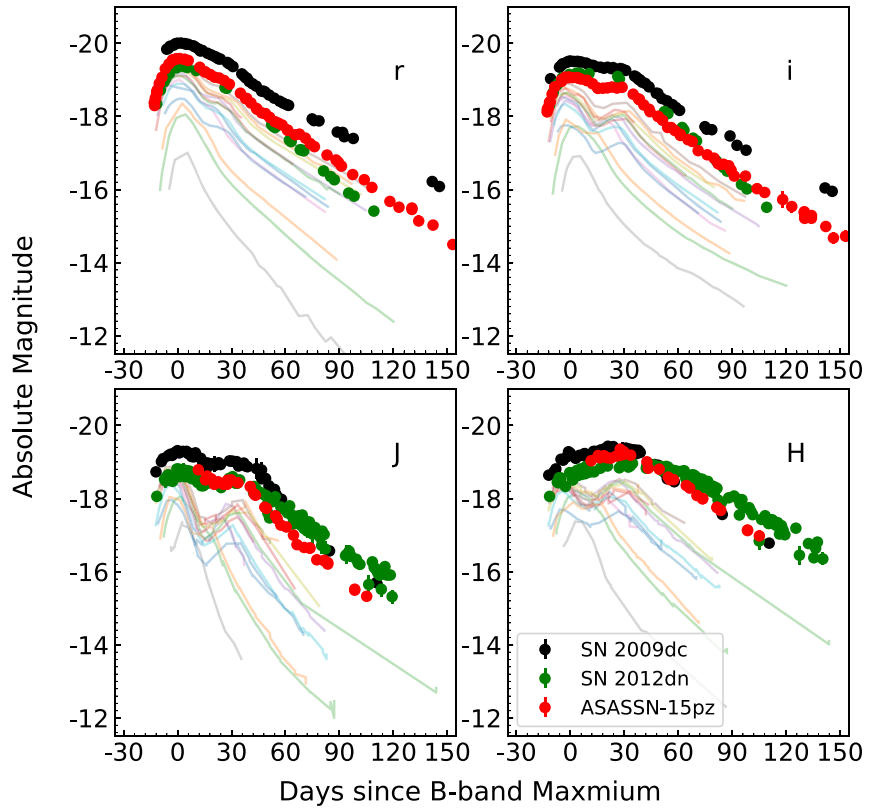

Figure 6. Absolute optical ( $r i)$ and NIR $(J H)$ light curves of ASASSN-15pz compared to those of SN 2012dn (Yamanaka et al. 2016), SN 2009dc (Taubenberger et al. 2011; Friedman et al. 2015), and a sample selected from the literature (Friedman et al. 2015; Krisciunas et al. 2017).

SN 2009dc, and SN 2012dn are bluer in the UV than the SNe Ia in our comparison sample, suggesting a higher blackbody temperature or less Fe line blanketing for these objects.

\subsection{Reddening and Luminosity}

The Galactic extinction toward ASASSN-15pz is only $E(B-V)_{\mathrm{MW}}=0.016$ mag based on the Schlafly \& Finkbeiner (2011) recalibration of the Schlegel et al. (1998) dust map. The low Galactic extinction is consistent with the absence of $\mathrm{Na} I \mathrm{D}$ absorption lines in all our spectra. NaiD absorption is not detected at the host-galaxy redshift, with a $3 \sigma$ upper limit on the equivalent width of EW(Na I D) $<0.2 \AA$. This corresponds to a $1 \sigma$ upper limit of $E(B-V)<0.04$ mag based on Phillips et al. (2013).

Based on this nondetection of $\mathrm{Na}$ I D, we assume that the host-extinction correction for ASASSN-15pz is negligible. The reddening-free light curve of ASASSN-15pz makes it uniquely valuable to understand the intrinsic color and luminosity of luminous SNe-Ia-pec. As discussed in Section 3.1 and shown in Figure 5, the post-peak $(B-V)$ curve of SN 2009dc has a nearly identical slope to ASASSN-15pz $\left(s_{1}[2009 \mathrm{dc}]=\right.$ $-0.0045 \pm 0.0005$ and $s_{1}[$ ASASSN $-15 \mathrm{pz}]=-0.0040 \pm$ $0.0002)$. In light of this and the close spectroscopic similarities between them, SN 2009dc and ASASSN-15pz likely have the same intrinsic color, which means that the host extinction for SN 2009dc is $E(B-V)_{\text {host }}=0.12 \pm 0.02 \mathrm{mag}$ in addition to the Galactic extinction of $E(B-V)_{\mathrm{MW}}=0.062 \mathrm{mag}$

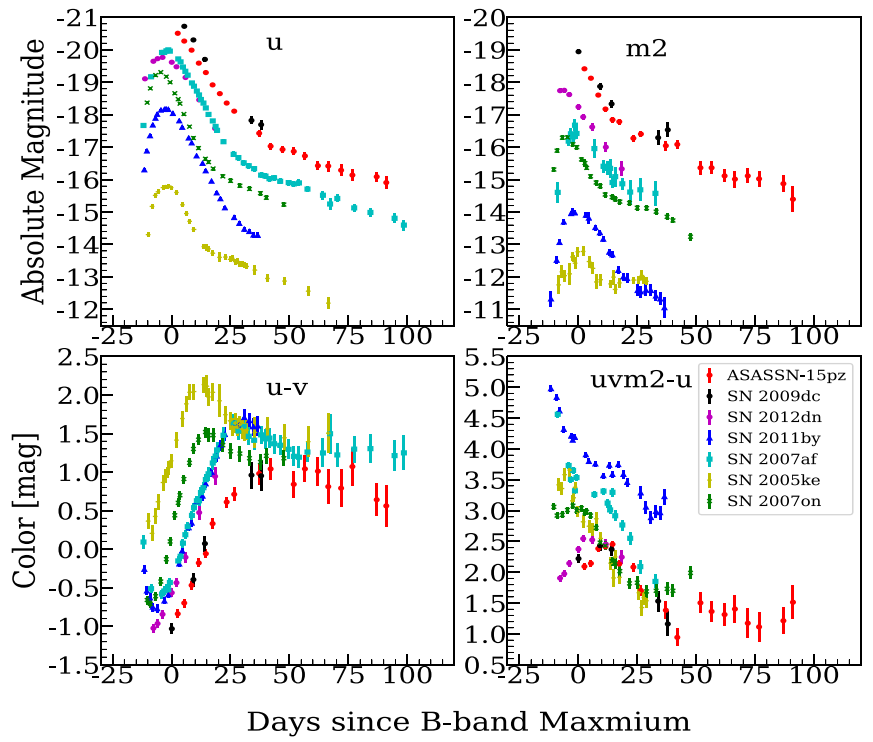

Figure 7. Swift UVOT light curves of ASASSN-15pz compared to a sample of selected 2009dc-like SNe and SNe Ia with good UV coverage. Galactic and host-galaxy extinction corrections are made for all targets. The top two panels show the absolute-magnitude light curves in the $u$ and $m 2$ bands. The bottom two panels show the color evolution of $(u-v)$ and $(u v m 2-u)$, which exhibit significant diversity.

(Schlafly \& Finkbeiner 2011) for a total extinction of $E(B-V)_{\text {tot }}=0.18 \mathrm{mag}$.

Silverman et al. (2011) obtained EW(Na I D) $=0.94 \pm 0.15 \AA$ for the host galaxy of SN 2009dc, which is consistent with the EW(Na I D) $\approx 1.0 \AA$ derived by Taubenberger et al. (2011). We note that according to the relation derived by Poznanski et al. (2012), $\mathrm{EW}(\mathrm{Na}$ I D) $=0.94 \pm 0.15 \AA$ translates into $E(B-V)_{\text {host }}=0.18_{-0.07}^{+0.10} \mathrm{mag}$, which is consistent with our determination.

There is also non-negligible host-galaxy extinction for SN 2006gz and SN 2012dn, with EW(Na ID)[2012dn] $\approx 0.75 \AA$ (Chakradhari et al. 2014) and EW(Na I D)[2006gz] $\approx 0.3 \AA$ (Hicken et al. 2007), respectively. For both SNe, the post-peak $(B-V)$ decline rates are much steeper than ASASSN-15pz, thus we cannot derive their host-reddening from a direct color curve comparison with ASASSN-15pz. Hicken et al. (2007) found that the post-peak (between 35 days and 51 days) $(B-V)$ decline rate of SN 2006gz was similar to $\mathrm{SNe} \mathrm{Ia}$, and by applying the Lira relation, they derived $E(B-V)=0.18 \pm 0.05 \mathrm{mag}$, which was consistent with the limit of $E(B-V)_{\text {host }} \leqslant 0.15 \pm 0.08 \mathrm{mag}$ that they estimated using $\mathrm{EW}(\mathrm{NaID})$. However, there is no evidence supporting the idea that the intrinsic color evolution of luminous SNe Iapec follow that of normal SNe Ia. In fact, Chakradhari et al. (2014) found that the Lira relations would yield $E(B-V)_{\text {Lira }} \approx 0.43 \mathrm{mag}$ for SN $2012 \mathrm{dn}$, which would be much higher than the $E(B-V)_{\text {host }} \approx 0.12$ mag estimated 
Table 3

Basic Comparison between 2009dc-like SNe Ia and SN 1991T

\begin{tabular}{|c|c|c|c|c|c|c|}
\hline SN & $z$ & $\mu(\mathrm{mag})$ & $E(B-V)_{\mathrm{MW}}(\mathrm{mag})$ & $E(B-V)_{\text {host }}(\mathrm{mag})$ & $M_{B}^{\text {peak }}(\mathrm{mag})$ & $M_{V}^{\text {peak }}(\mathrm{mag})$ \\
\hline ASASSN-15pz & 0.014837 & 33.85 & 0.016 & 0.0 & -19.69 & -19.67 \\
\hline $2012 \mathrm{dn}$ & 0.010187 & 33.14 & 0.06 & 0.12 & -19.51 & -19.40 \\
\hline $2009 \mathrm{dc}$ & 0.021391 & 34.85 & 0.06 & 0.12 & -20.26 & -20.22 \\
\hline $1991 \mathrm{~T}$ & 0.00579 & 30.74 & 0.019 & 0.15 & -19.73 & -19.74 \\
\hline $2005 \mathrm{M}$ & 0.024624 & 35.09 & 0.027 & 0.06 & -19.45 & -19.36 \\
\hline
\end{tabular}

from the NaiD absorption. Because we have no extinction-free analogs of SN $2006 \mathrm{gz}$ or SN 2012dn, it is not safe to derive their host-reddening by making assumptions on their intrinsic color evolutions. We adopt extinction estimates derived from the NaiD absorption for SN 2006gz and SN 2012dn in our analysis.

The adopted extinction and peak luminosities for these four luminous SNe Ia-pec are given in Table 3, which also includes the parameters for SN 1991T (Phillips et al. 1999). The peak luminosity of ASASSN-15pz is very similar to SN $1991 \mathrm{~T}$ but $\approx 0.6$ mag dimmer than SN 2009dc.

\subsection{Bolometric Light Curve}

To construct a bolometric light curve we first convert the observed UV, optical, and NIR magnitudes into monochromatic fluxes and de-redshift them into the rest frame. We use only the $u v m 2$ - and $u$-band Swift data because of the red-leak issue in the $u v w 2$ and $u v w 1$ filters (Brown et al. 2010). The resulting spectral energy distributions (SEDs) are shown in Figure 8, where the peak wavelength changes gradually from blue to red as the ejecta expand and cool. Since the UV and NIR observations started about 2 days and 10 days after peak, respectively, we extrapolate our early-phase UV and NIR light curve to construct the bolometric light curves using the shapes of the UV and NIR light curves of SN 2012dn and SN 2009dc, respectively. We integrate the SEDs to construct pseudobolometric ("uvoir+") light curves covering from 2000 to $23550 \AA$ A. We also construct pseudo-bolometric ("uvoir") light curves covering 3100-23550 $\mathrm{A}$, in order to make direct comparisons with those for SN 2009dc and SN 2012dn (see Figure 9). These pseudo-bolometric light curves of SN 2009dc and SN 2012dn are constructed using the same method as for ASASSN-15pz.

For SNe Ia, the flux at wavelengths blueward of $3100 \AA$, is generally believed to make a small contribution to the bolometric flux (see, e.g., Contardo et al. 2000; Contreras et al. 2018). But SN 2009dc and SN 2012dn are exceptionally blue (Brown et al. 2014a) and the UV contribution to their bolometric luminosity near the peak is significant. Similarly, as shown in the bottom two panels of Figure 9, we find that for ASASSN-15pz, there is significant UV contribution to the bolometric light, and the U/uvoir (MUV/uvoir) fraction evolves from as high as $\sim 45 \%(\sim 23 \%)$ at $-12 \mathrm{~d}$ to $\sim 38 \%$ $(\sim 15 \%)$ at the $B$-band peak, and then to a constant level of $\sim 10 \%(\sim 6 \%)$ for +30 days and the later phases. The earlytime UV and late-time NIR contributions show considerable diversities reflecting the diversities seen in the color evolution discussed in Sections 3.2 and 3.1. This makes it important to have UV and NIR data to construct a reliable bolometric light curve rather than assuming the fractions observed from some template objects.

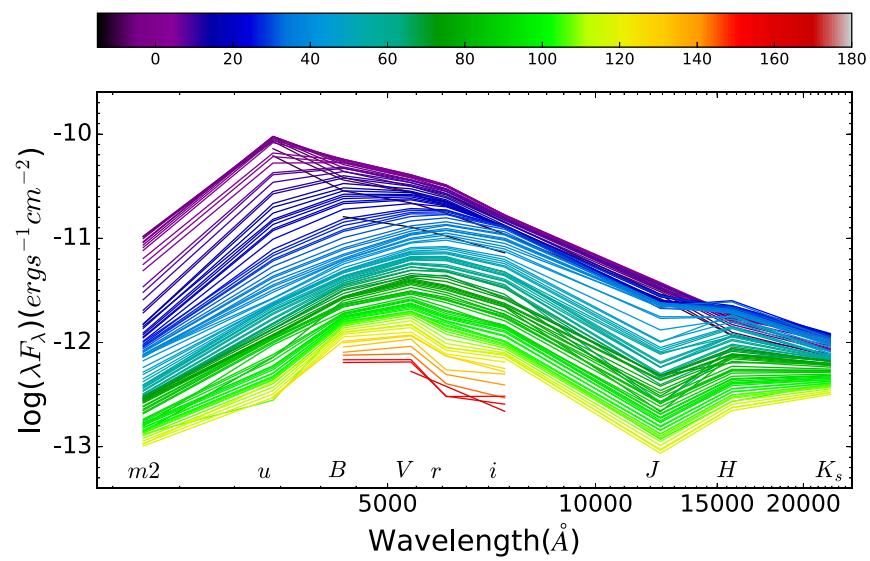

Figure 8. Rest-frame SEDs of ASASSN-15pz. The colors indicate the phase relative to the $B$-band maximum as indicated by the color bar.

\subsection{Mass of ${ }^{56} \mathrm{Ni}$}

$\mathrm{SNe}$ Ia are powered by the radioactive decay of ${ }^{56} \mathrm{Ni}$ synthesized in the thermonuclear explosion, and the mass of ${ }^{56} \mathrm{Ni}$ is a key physical parameter. We can approximately estimate the mass of ${ }^{56} \mathrm{Ni}$ using "Arnett's rule" (Arnett 1982) that $L_{\text {bol }}^{\text {peak }} \approx \alpha Q\left(t_{\text {peak }}\right) M_{\mathrm{Ni}}$, where $Q\left(t_{\text {peak }}\right)$ is the rate of radioactive energy production per unit nickel mass at time of peak luminosity after the explosion, $\alpha$ is a coefficient of order unity and $L_{\mathrm{bol}}^{\mathrm{peak}}$ is the peak bolometric luminosity. The ASASSN detection on UT 2015 September 27.16 means that $t_{\text {peak }}>14.6$ days. If we fit our early light curve (phase $<-10$ days) to a fiducial fireball model $f=A\left(t+t_{R}\right)^{2}$ we get a best-fit estimate of $t_{\text {peak }}=21.4$ days. For comparison, Silverman et al. (2011) obtained a direct constraint on $t_{\text {peak }}$ for SN 2009dc from early detections as $t_{\text {peak }}>21$ days, and combining with non-detections they estimated a rise time of 23 with an uncertainty of 2 days. The ${ }^{56} \mathrm{Ni}$ mass estimate using "Arnett's rule" is sensitive to the estimate of explosion time, and we adopt an uncertainty of 2 days similar to Silverman et al. (2011). With $L_{\text {bol, peak }}=2.7 \times 10^{43} \mathrm{erg} \mathrm{s}^{-1}$ for ASASSN-15pz, we obtain $M^{56 \mathrm{Ni}} \approx 1.1 \pm 0.4 M_{\odot}$ with $t_{\text {peak }}=21.4 \pm 2$ days and $\alpha=1.0 \pm 0.2$ (see, e.g., Branch 1992; Stritzinger \& Leibundgut 2005, for various estimates of $\alpha$ ).

The radioactive-decay energy is principally released as $\gamma$-rays and positrons. At late times, the fraction of $\gamma$-ray energy deposited in the ejecta is given by $t_{0}^{2} / t^{2}$, where $t_{0}$ is the $\gamma$-ray escape timescale (e.g., Jeffery 1999; Stritzinger et al. 2006). For $t \ll t_{0}$, all gamma-rays are trapped and the deposition fraction is unity. To a good approximation, the deposition can be interpolated by $1-\exp \left(-t_{0}^{2} / t^{2}\right)$ at all times (see, e.g., Wygoda et al. 2019a). As shown by Katz et al. (2013), at sufficient late times, there are two relations between the 


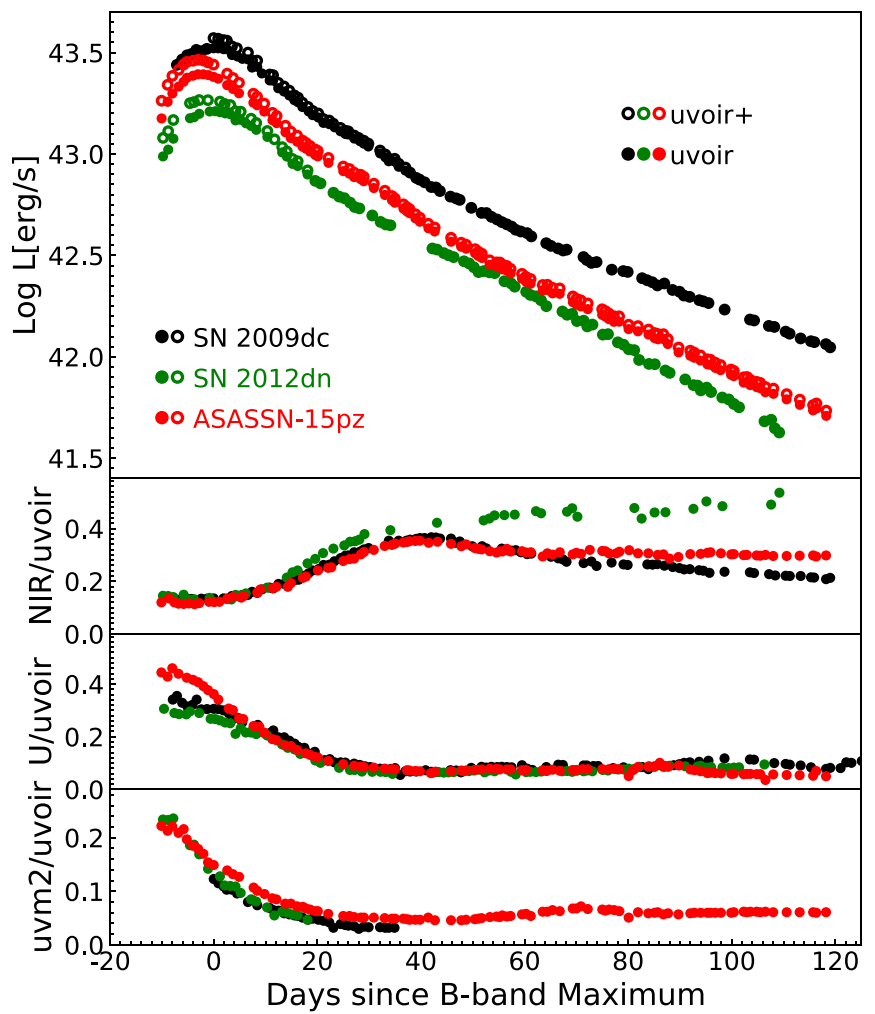

Figure 9. Bolometric light curve and fractional contributions from different wavelength ranges to ASASSN-15pz, SN 2009dc, and SN 2012dn. In the uppermost panel, the uvoir bolometric light curves shown in filled circles (ASASSN-15pz in red, SN 2009dc in black, and SN 2012dn in green) are constructed from UVOT $u$ (or ground-based $U$ band), BVri(RI), and $J H K s$ bands photometry data, while the uvoir + bolometric lights using empty circles include UVOT $u v m 2$-band flux. The bottom three panels show the fractional contributions from the NIR (from 8150 to $23550 \AA$ ), $U$ (from 3100 to $3930 \AA$ ), and $u v m 2$ (from 2000 to $3000 \AA$ ) bands relative to the uvoir luminosity.

deposition and the bolometric luminosity, $Q(t)=L(t)$ and the integral $\int_{0}^{t} Q\left(t^{\prime}\right) t^{\prime} d t^{\prime}=\int_{0}^{t} L_{\mathrm{bol}}\left(t^{\prime}\right) t^{\prime} d t^{\prime}$, and the ${ }^{56} \mathrm{Ni}$ mass can be derived using these relations (see some examples by Blondin et al. 2018; Wygoda et al. 2019a). For ASASSN-15pz, we first obtain $t_{0}=46.4 \pm 0.5$ days by fitting $t^{2} L / \int L t d t$, which is independent of ${ }^{56} \mathrm{Ni}$ mass, to the observed bolometric light from 60 days to 115 days after explosion, as shown in bottom panel of Figure 10. Then we fit the integral $\int L t d t$, as shown in the middle panel, and obtain $M^{56 \mathrm{Ni}}=1.13 \pm 0.14 M_{\odot}$. Unlike Arnett's rule, the estimate of $M_{56} \mathrm{Ni}$ with the Katz et al. (2013) integral method is insensitive to the exact value of $t_{\text {peak }}$, as the integral $\int L t d t$ has little weight from the early phases. The $\gamma$-ray escape timescale $t_{0}$ depends on the ${ }^{56} \mathrm{Ni}$ mass weighted column density of the ejecta (see, e.g., Jeffery 1999; Wygoda et al. 2019a), and $t_{0}=46.4 \pm 0.5 \mathrm{~d}$ for ASASSN-15pz is higher than almost all SNe Ia in the sample analyzed by Wygoda et al. (2019a), suggesting that it has a relatively high ejecta column density. The same method applied to our bolometric light curve for SN 2009dc yields $t_{0}=55.1 \pm 1.0$ days and $M^{56} \mathrm{Ni}=1.6 \pm 0.1 M_{\odot}$, implying higher ejecta column density and $M^{56} \mathrm{Ni}$ than ASASSN-15pz.

The broad consistency between the two ${ }^{56} \mathrm{Ni}$ mass estimates is similar to that found for $\mathrm{SNe}$ Ia and supports radioactive decay as the energy source in these objects over alternative scenarios such as ejecta-CSM interaction (see, e.g., Noebauer et al. 2016).
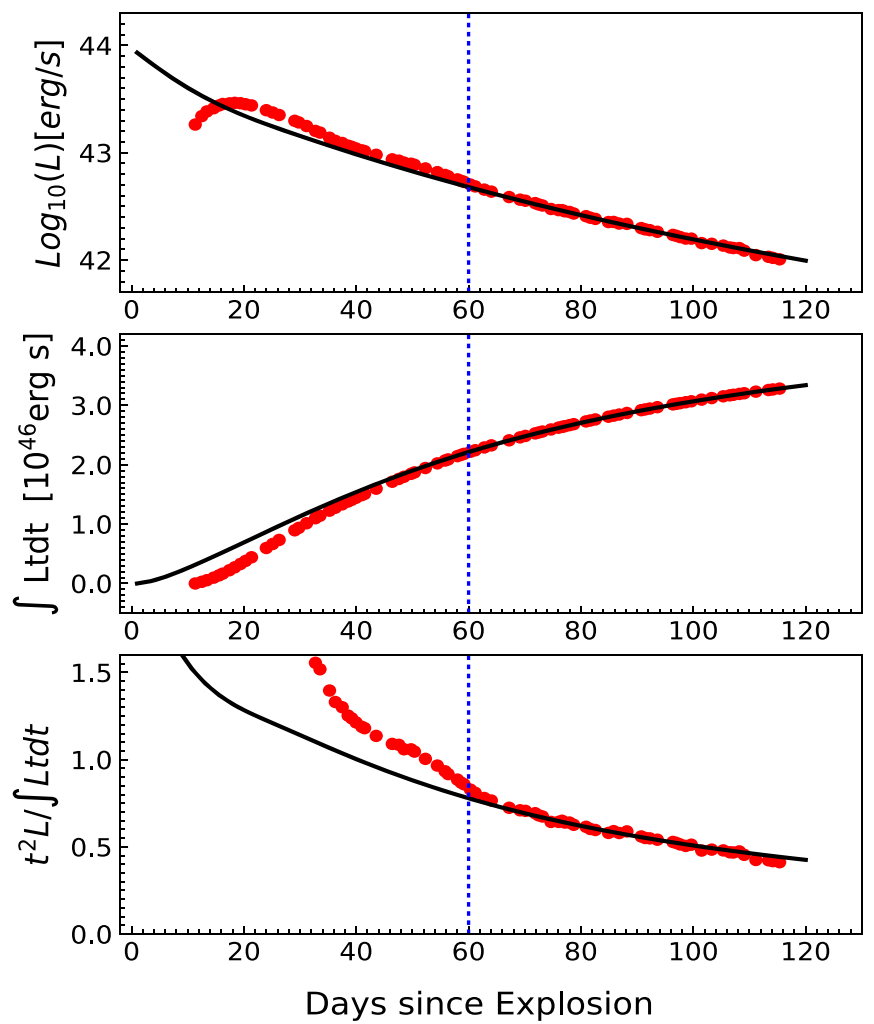

Figure 10. Model fit (black solid line) to the observed bolometric light curve of ASASSN-15pz. The bottom panel shows the fitting result to $t^{2} L / \int L t d t$ and the middle panels show the fitting result to the integral $\int L t d t$. The black line in the upper panel represents the energy deposition of the gamma-rays and positrons produced by the decay of ${ }^{56} \mathrm{Ni}$ for the best-fit ${ }^{56} \mathrm{Ni}$ mass and $\gamma$-ray escape timescale $t_{0}$. The dotted lines indicate the phase (60 days) after which the data are used for the fits.

\section{Optical Spectra}

In Figure 11, we compare rest-frame spectra for ASASSN$15 \mathrm{pz}$ ranging from -11.6 days to +240 days with those of SN 2009dc at similar phases. The spectra are extinctioncorrected. At all phases, the spectral features of ASASSN-15pz are almost identical to SN 2009dc: ASASSN-15pz is a spectroscopic twin of SN 2009dc. In the following sections, we discuss the spectroscopic features at various phases.

\subsection{Pre-maximum Spectrum}

Using the earliest spectrum shown in Figure 11, Taddia \& Stritzinger (2015) reported their spectroscopic classification of ASASSN-15pz as a SN Ia at a phase near maximum. There is a prominent C II absorption feature redward of the Si II $\lambda 6355$ feature, distinguishing it from pre-peak spectra of the Type Ia SNe 2003du (Stanishev et al. 2007) and 1991T (Filippenko et al. 1992b; Jeffery et al. 1992; Ruiz-Lapuente et al. 1992; Mazzali et al. 1995), as shown in Figure 12. This strong C II feature appears to be a unique signature of 2009dc-like objects at early phases (e.g., SN 2009dc from Taubenberger et al. 2011; SN 2006gz from Hicken et al. 2007; SN 2012dn from Parrent et al. 2016).

The C II absorption feature is commonly interpreted as being due to unburned carbon, and the C II species can be seen at wavelengths of $4267,4745,6580$ and $7234 \AA$ in the optical spectra (see, e.g., Mazzali 2001). Folatelli et al. (2012) found that at least $30 \%$ of CSP-I SNe Ia exhibit C II absorption at 


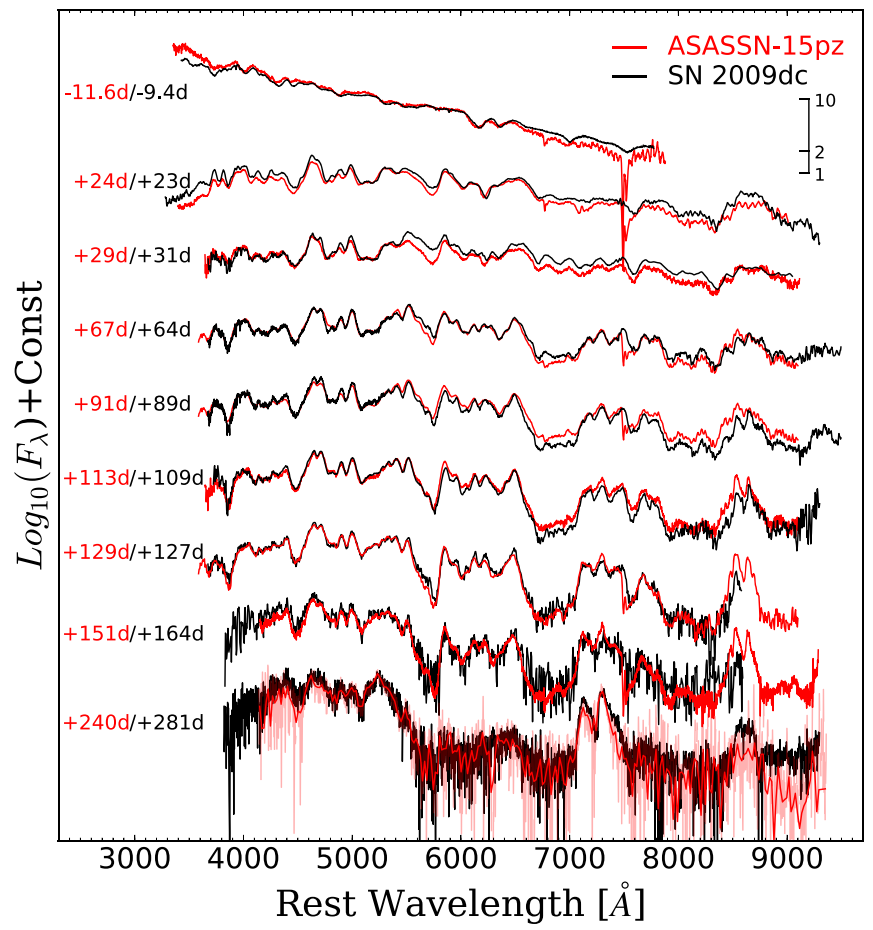

Figure 11. Spectra of ASASSN-15pz (red) compared to those of SN 2009dc (black), revealing that they are spectroscopic twins from pre-peak to nebular phases (phases are labeled at the left). The $Y$-axis is on logarithmic scale, and the vertical bar on the upper right shows the scale for factors of 2 and 10. All spectra have been shifted to the rest frame, and the median fluxes of SN 2009dc's spectra between 5000 and $5500 \AA$ have been normalized to match those of ASASSN-15pz at the corresponding epochs. All reduced spectra are made available at the Weizmann Interactive Supernova Data Repository (WISeREP; Yaron \& Gal-Yam 2012).

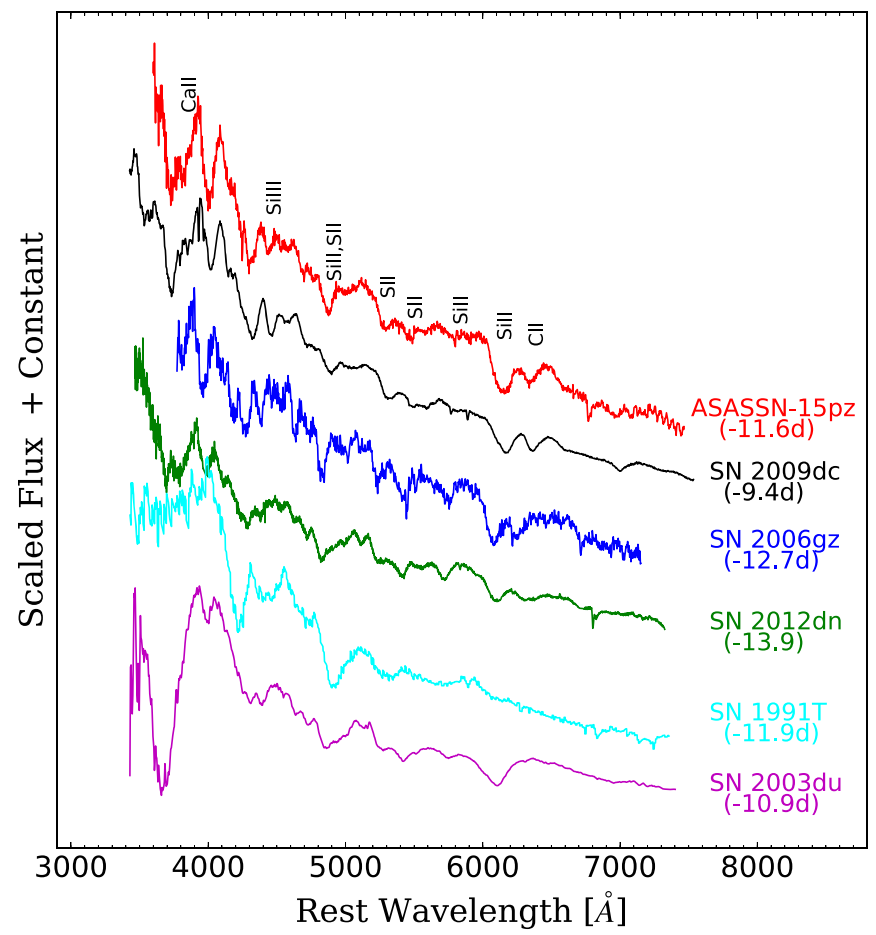

Figure 12. Pre-maximum spectrum of ASASSN-15pz compared with various $\mathrm{SNe}$ Ia at similar phase (in parentheses). From top to bottom, the $\mathrm{SNe}$ are overluminous SN 2009dc (Taubenberger et al. 2011), SN 2006gz (Hicken et al. 2007), SN 2012dn (Parrent et al. 2016), the over-luminous prototype SN 1991 T (Mazzali et al. 1995), and the normal SN 2003du.

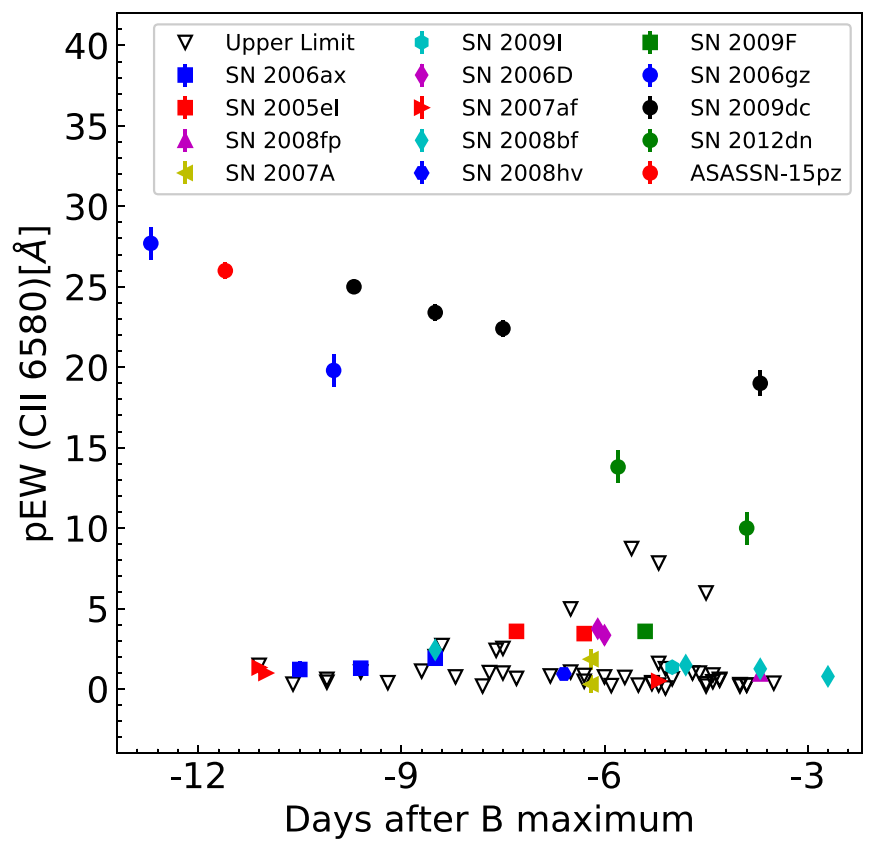

Figure 13. Pseudo-equivalent width of $\mathrm{C}$ II $\lambda 6580$ in ASASSN-15pz as compared to SN 2009dc, SN 2006gz, SN 2012dn, and other SNe Ia from the CSP-I sample (Folatelli et al. 2012).

$\lambda 6580$, and a similar fraction was reported by Parrent et al. (2011). The C II feature is unusually strong in 2009dc-like objects. This is shown in Figure 13, where the pseudoequivalent width of C II $\lambda 6580$ of ASASSN-15pz is shown along with those of SN 2006gz, SN 2009dc, and SN 2012dn in comparison to SNe Ia from the CSP sample in Folatelli et al. (2012). The absorption strengths of 2009dc-like objects appear to separate them from the CSP SNe Ia.

We measure expansion velocities of $9850 \mathrm{~km} \mathrm{~s}^{-1}$ and $10900 \mathrm{~km} \mathrm{~s}^{-1}$ from the Si II $\lambda 6355$ and C II $\lambda 6580$ absorption lines in the -11.6 days spectrum. The expansion velocities of ASASSN-15pz are generally $\sim 1000 \mathrm{~km} \mathrm{~s}^{-1}$ higher than SN 2009dc at similar phases but they are substantially lower than normal SNe Ia, as illustrated by SN 2002bo (Benetti et al. 2004), SN 2003du (Stanishev et al. 2007), SN 2005cf (Wang et al. 2009), and SN 2006X (Yamanaka et al. 2009b) in Figure 14. The slowly expanding ejecta are also a well known feature of the 2009dc-like SNe.

\subsection{One and Two Months after Maximum}

In Figure 15, we compare the spectra of ASASSN-15pz one and two months after peak with those of the peculiar SN Ia 2002cx (Li et al. 2003), SNe Ia SN 2003du (Stanishev et al. 2007) and SN 2005cf (Wang et al. 2009), SNe Ia-pec SN 2009dc (Taubenberger et al. 2011), and SN 2012dn (Childress et al. 2016; Yamanaka et al. 2016) at similar phases. All pronounced line features in SNe Ia (here SN 2003du and SN $2005 \mathrm{cf}$ ) are present in ASASSN-15pz, but the features for ASASSN-15pz (like 2009dc) are narrower and sharper. Si II $\lambda$ 6355 absorption was detected in the +64 days spectrum of SN $2009 \mathrm{dc}$, and it is tentatively detected in the +67 days spectrum of ASASSN-15pz, while this feature is usually not seen in $\mathrm{SNe}$ Ia at such late phases. Silverman et al. (2011) attributed such strong and long-lived Si II $\lambda 6355$ absorption in SN 2009dc to synthesizing more silicon in the explosion than a typical SN Ia. 


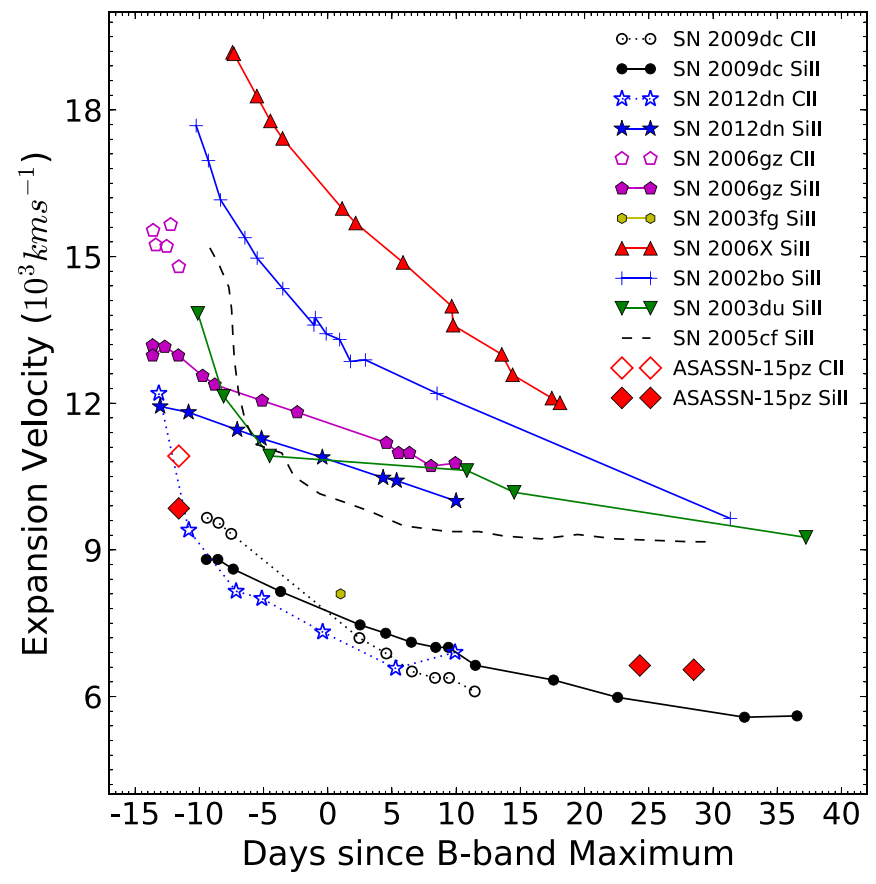

Figure 14. Spectral velocity evolution of the Si II $\lambda 6355$ absorption line for ASASSN-15pz as compared with those of SNe Ia-pec (SN 2009dc, SN 2012dn, SN 2006gz, SN 2007if, 2003fg), and SNe Ia (SN 2006X, SN 2002bo, SN 2003du and SN 2005cf). C II $\lambda 6580$ velocities are shown for ASASSN$15 \mathrm{pz}, \mathrm{SN} 2009 \mathrm{dc}, \mathrm{SN} 2006 \mathrm{gz}$, and SN 2012dn.

At 1-2 months, the spectra of SN 2012dn have line shapes and widths more similar to normal SNe Ia than SN 2009dc/ ASASSN-15pz. The differences between SN 2012dn and SN 2009dc/ASASSN-15pz in the spectra at 1-2 months are likely due to the higher ejecta velocities, as shown in Figure 14. The ejecta velocity of SN 2012dn derived from Si II $\lambda 6355$ is $\sim 4000 \mathrm{~km} \mathrm{~s}^{-1}$ higher than SN 2009dc or $\sim 3000 \mathrm{~km} \mathrm{~s}^{-1}$ higher than ASASSN-15pz. Silverman et al. (2011) noted the interesting similarities in spectral features and line widths between SN 2009dc and SN 2002cx at these phases. One notable difference is the absence of Si II $\lambda 6355$ absorption in SN 2002cx, while it is relatively strong for SN 2009dc/ ASASSN-15pz. As we discuss in Section 4.3, the later-phase spectra of ASASSN-15pz/SN 2009dc are substantially different from SN 2002cx.

\subsection{Nebular-phase Spectra}

The supernova ejecta become (nearly) optically thin a few months after explosion. These Nebular-phase spectra probe the inner regions of the ejecta, often providing key insights into the explosion and clearly distinguishing different classes of supernovae. We show comparisons of the late-time spectra of ASASSN-15pz $(+129$ days and +240 days, respectively) with $\mathrm{SNe}$ Ia and Ia-like peculiar objects at similar phases in Figure 16.

We label the prominent forbidden emission lines that shape the general nebular phase spectra of $\mathrm{SNe}$ Ia (see, e.g., Axelrod 1980; Maguire et al. 2018) in Figure 16. The main $\mathrm{Fe}$ and Co features between $\sim 4000 \AA$ and $\sim 8000 \AA$, are present in all sub-classes of SNe Ia but exhibit a broad range of line widths and velocity distributions (see, e.g., Mazzali et al. 1998; Kushnir et al. 2013; Dong et al. 2015). The nebular spectra of ASASSN-15pz and SN 2009dc are well distinguished from normal SNe Ia. In spite of the spectroscopic differences from SN 2009dc at 1-2 months, the late-time spectrum ( +115 days) of SN 2012dn closely resembles those of SN 2009dc and ASASSN-15pz, providing good evidence that SN 2012dn is a member of the 2009dc-like SNe Ia-pec class. Similarly, as shown in Figure 16, the nebular spectra of SN 2007if, especially the nebular spectrum at +384 days, is more similar to the 2009 dc-like spectra than to those of the normal SNe Ia.

At $~ 4-5$ months (left panel of Figure 16), the differences between 2009dc-like SNe Ia and SNe Ia (SN 2003du and SN 1991T) are clearly seen in at least the following three aspects: (1) the nebular features of 2009dc-like SNe are generally sharper (i.e., lower velocities), and there are usually additional narrow features at the location of the main $\mathrm{Fe}$ or $\mathrm{Co}$ features of $\mathrm{SNe} \mathrm{Ia}$ (e.g., the prominent $[\mathrm{Fe}$ II $] /[\mathrm{Fe}$ III] complex between $4400 \AA$ and $5700 \AA$ with two main broad peaks and also the [Co III] region to their red); (2) the feature around $7300 \AA$ (likely including contributions from [Ca II] $\lambda 7291, \lambda 7324$, and [Fe II] $\lambda 7155)$ is relatively stronger than in SN 2003du and SN 1991T, and there is an additional emission feature at $7660 \AA$; (3) strong emission lines at around $8600 \AA$ (Ca triplet) are seen in ASASSN-15pz and SN 2012dn. These are are commonly seen in the nebular phase spectra of core-collapse supernovae, but not seen in SN 2003du and SN 1991 T at similar phases (Maguire et al. 2018). Detailed modeling will be needed to understand whether the presence of strong Ca II triplet nebular lines is compatible with models synthesizing a large of amount of ${ }^{56} \mathrm{Ni}$.

In nebular-phase spectra obtained beyond 200 days after $B_{\max }$, 2009dc-like SNe Ia, including ASASSN-15pz, are clearly distinguished from any other class of SNe Ia or Ia-like objects (from 1991bg-like SN Ia to 1991T-like SNe Ia also 2002cx-like SN Ia-pec) as shown in the right panel of Figure 16. Thus, even if the very early-phase spectra are missing, it is possible to distinguish 2009dc-like SNe from SNe Ia and other Ia-like SNe. Similar to SNe Ia, the blue part of the optical spectra of ASASSN-15pz and other 2009dc-like objects are dominated by $[\mathrm{Fe}$ II $] /[\mathrm{Fe}$ III] complexes between $4400 \AA$ and $5700 \AA$ but their shapes and line ratios are substantially different. As pointed out by Taubenberger et al. (2013), the $5200 \AA$ feature, a blend of [Fe II] and [Fe III], is stronger in 2009dc-like SNe Ia than the features at $4700 \AA$ that are primarily due to [Fe III], implying a higher [Fe II] fraction and a lower ionization state in the ejecta than normal SNe Ia. The higher [Fe II] to [Fe III] ratio is also supported by the relatively strong feature at around $4300 \AA$, which is attributed to emission of [Fe II] $\lambda 4244$ and [Fe II] $\lambda 4416$ (Mazzali \& Hachinger 2012; Mazzali et al. 2015). Taubenberger et al. (2013) suggested that the lower ionization states found in 2009dc-like SNe Ia might be the result of enhanced recombination due to having higher inner ejecta densities due to the low expansion velocities of the 2009 dc-like objects.

\section{Summary and Discussion}

Our comprehensive photometric and spectroscopic data of ASASSN-15pz add to a handful of well-studied 2009dc-like objects. The main results of our analysis are as follows:

1. ASASSN-15pz is a spectroscopic twin with the luminous SN Ia-pec SN 2009dc at all observed phases, and they share many common properties that distinguish them from the SNe Ia population, such as being exceptionally UV bright at early phases, showing strong C II $\lambda 6580$ 


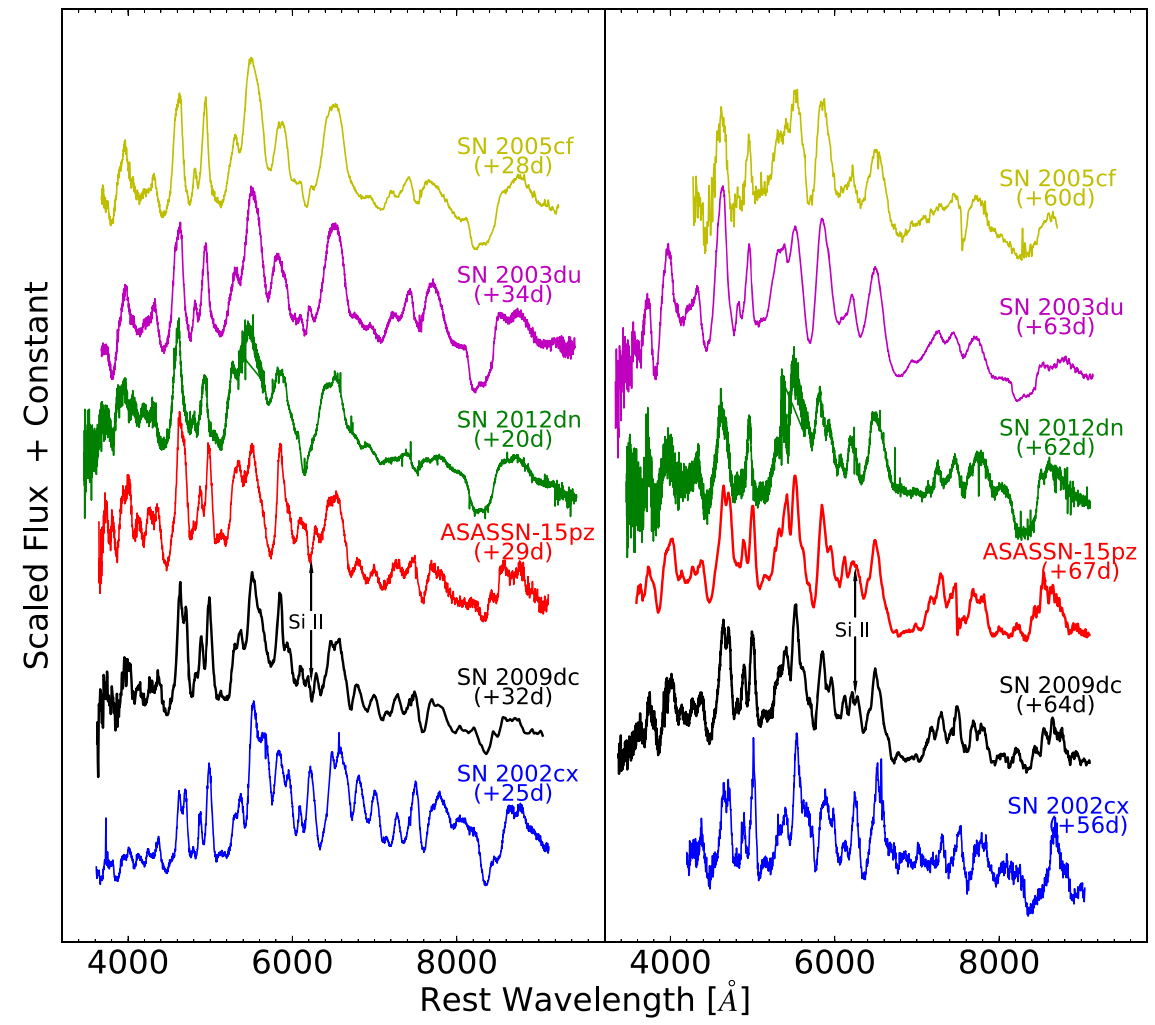

Figure 15. Spectra of ASASSN-15pz at phase +28.5 days (in left panel) and phase +67 days (in right panel) are compared with those of SN $2012 \mathrm{dn}$, SN $2009 \mathrm{dc}$, SN $2002 \mathrm{cx}, \mathrm{SN} 2005 \mathrm{cf}$, and SN 2003du. The phases relative to maximum light are indicated for each spectrum (in parentheses).

absorption at pre- and near-peak spectra, and having relatively slow expansion velocities.

2. With little host extinction, the peak luminosity of ASASSN$15 \mathrm{pz}$ is well determined $\left(M_{B}^{\text {peak }}=-19.69 \pm 0.12 \mathrm{mag}\right.$ and $\left.M_{V}^{\text {peak }}=-19.67 \pm 0.12 \mathrm{mag}\right)$, which is comparable with 1991T-like objects at the luminous end of SN Ia luminosity function but substantially dimmer than SN 2009dc.

3. From the bolometric light curve, the ${ }^{56} \mathrm{Ni}$ mass synthesized in the explosion of ASASSN-15pz is $1.13 \pm 0.14 M_{\odot}$, which is more than all normal SNe Ia but lower than that found for several 2009dc-like SNe Iapec. The $\gamma$-ray escape timescale of $t_{0}=46.4 \pm 0.5$ days is considerably higher than those of normal SNe Ia, implying a relatively high ejecta column density.

4. ASASSN-15pz has broad $B$ - and $V$-band light curves and its post-peak decline rate of $m_{15}(B)=0.67 \pm 0.07 \mathrm{mag}$ is similar to $\mathrm{SN} 2009 \mathrm{dc}$ and slower than all normal SNe Ia. As a population, 2009dc-like $\mathrm{SNe}$ show a significant diversity in peak luminosities and do not follow the tight width-luminosity relation (Phillips 1993; Burns et al. 2014, 2018) of the SN Ia population. The NIR and late-time $\gtrsim 50$ days optical light curves of the three well-studied 2009dc-like objects (ASASSN-15pz, SN 2009dc and SN 2012dn) show large diversity in their evolutions.

We have collected $\sim 200$ objects in our complete volumelimited $(z<0.02)$ sample of SNe Ia and SNe Ia-like objects (P. Chen et al. 2019, in preparation). Of this sample ASASSN$15 \mathrm{pz}$ is the only $2009 \mathrm{dc}-$ like object, suggesting that the volumetric rate of $2009 \mathrm{dc}-$ like object is $\sim 1 \%$ of the total SN Ia rate. In the
100 type IA Supernovae (100IAS) survey (Dong et al. 2018), we systematically collect nebular phase spectra from our volumelimited sample. Nebular-phase spectra can be crucial for understanding explosion physics by probing the inner ejecta. In particular, the nebular-phase spectra of ASASSN-15pz and other 2009dc-like objects (including SN 2007if, SN 2009dc, and SN 2012dn) are well separated from normal SNe Ia, exhibiting characteristic features such as sharp nebular lines, low ionization states for $\mathrm{Fe}$ (low ratio of [ $\mathrm{Fe} \mathrm{II}]$ emission to [Fe III]), and strong [Ca II] lines and Ca II NIR triplet. Therefore, nebular spectra can provide definitive identification of $2009 \mathrm{dc}-$ like objects. The fact that 2009dc-like objects can exhibit significant diversity in their photometric properties even between spectroscopic twins may provide an avenue to constrain the progenitors or explosion process leading to these events.

The first few well-observed 2009dc-like SNe discovered since Howell et al. (2006) all reached high peak luminosities at $\sim-20 \mathrm{mag}$, leading to the speculation of super-Chandrasekharmass progenitors, although whether super-Chandrasekhar progenitors lead to 2009dc-like explosions is unknown (see, e.g., Hachinger et al. 2012; Noebauer et al. 2016; Fink et al. 2018 and also the discussion in Maoz et al. 2014). The popular naming, "Super-Chandrasekhar-mass SNe Ia," mainly rested upon the first 2009dc-like SNe being more luminous than all $\mathrm{SNe}$ Ia, whose origin were thought to be due to Chandrasekharmass explosions, according to popular theoretical notion. However, in recent years, there has been increasing observational and theoretical evidence against the Chandrasekhar-mass models as the main channel for the SN Ia population (e.g., see reviews by Maoz et al. 2014; Wang 2018). Here we find that 2009dc-like SNe Ia are not necessarily more luminous than all $\mathrm{SNe}$ Ia, which also undermines the empirical basis for the 


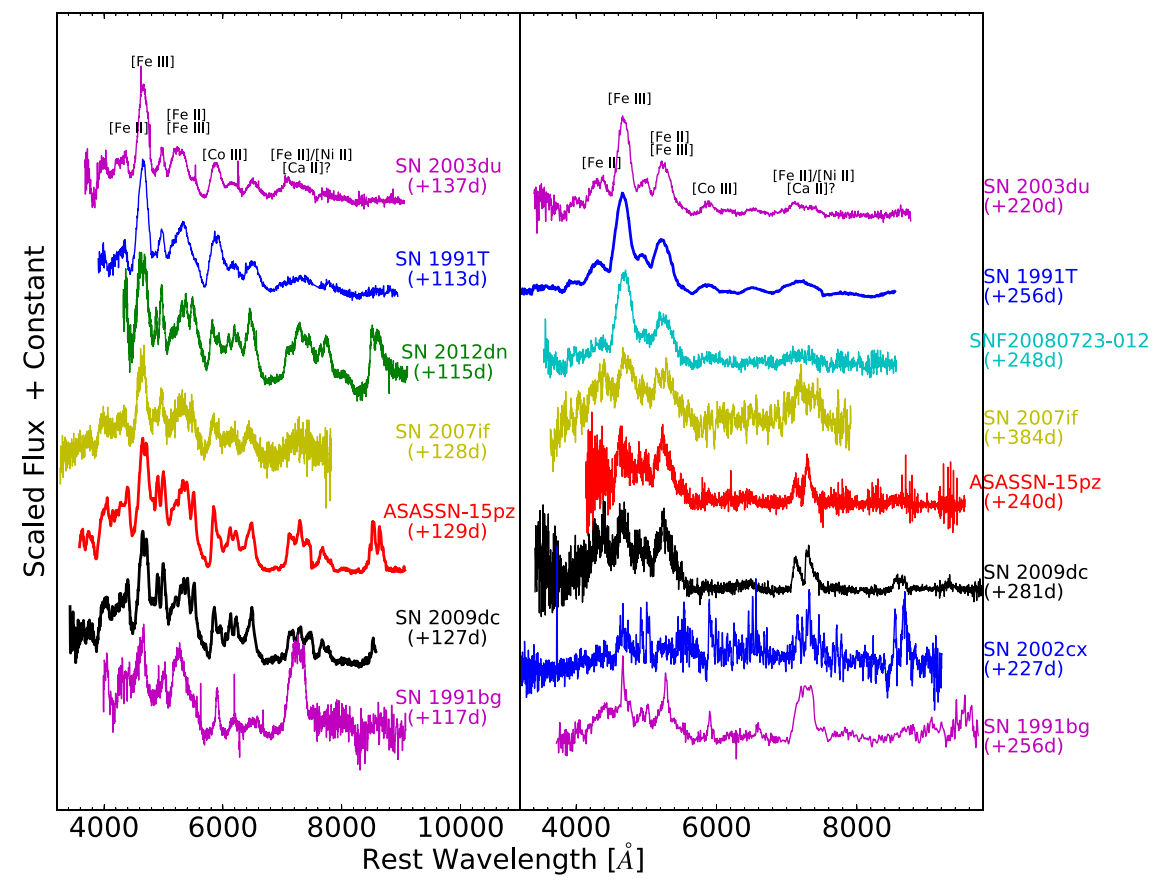

Figure 16. Left: early nebular-phase spectra of ASASSN-15pz compared with those of SNe Ia 2003du, SN 1991T, and SNe Ia-pec SN 2012dn (Parrent et al. 2016), SN 2007if (Blondin et al. 2012), and SN 2009dc. Right: nebular-phase spectra of ASASSN-15pz compared with those of SNe Ia 2003du (Stanishev et al. 2007), subluminous SN 1991bg (Turatto et al. 1996), over-luminous SN 1991T (Gómez and López 1998), 1991T-like SN Ia SNF20080723 (Scalzo et al. 2012 ), and SNe Ia-pec SN 2007if (Taubenberger et al. 2013), SN 2009dc (Silverman et al. 2011) as well as SN 2002cx (Jha et al. 2006).

"Super-Chandrasekhar" naming. We therefore suggest referring to them in the future as "2009dc-like SNe Ia-pec" to avoid using a designation that is based on an uncertain theoretical interpretation.

We thank Doron Kushnir, Erika K. Carlon, Francesco Taddia, Barry Madore, Mark Seibert, and Jeff Rich for their help. P.C., S.D., and S. B. acknowledge Project 11573003 supported by NSFC. This research uses data obtained through the Telescope Access Program (TAP), which has been funded by the National Astronomical Observatories of China, the Chinese Academy of Sciences, and the Special Fund for Astronomy from the Ministry of Finance. K.M. acknowledges support from STFC (ST/ M005348/1) and from H2020 through an ERC Starting Grant (758638). CSK, K.Z.S., and T.A.T. are supported by NSF grants AST-1515876, AST-1515927, and AST-1814440. P.J.B. is supported by NASA through HST program \#14144 through a grant from the Space Telescope Science Institute, which is operated by the Association of Universities for Research in Astronomy, Inc., under NASA contract NAS 5-26555. Support for J.L.P. is provided in part by FONDECYT through the grant 1191038 and by the Ministry of Economy, Development, and Tourism's Millennium Science Initiative through grant IC120009, awarded to The Millennium Institute of Astrophysics, MAS. This work is partly based on observations made with the Nordic Optical Telescope, operated by the Nordic Optical Telescope Scientific Association at the Observatorio del Roque de los Muchachos, La Palma, Spain, of the Instituto de Astrofisica de Canarias. The data presented here were obtained in part with ALFOSC, which is provided by the Instituto de Astrofisica de Andalucia (IAA) under a joint agreement with the University of Copenhagen and NOTSA. M.S. is supported in part by a generous grant (13261) from VILLUM FONDEN and a project grant from the Independent Research Fund Denmark. This work is based (in part) on observations collected at the European Organisation for
Astronomical Research in the Southern Hemisphere, Chile, under ESO programme 0101.D-0202, and as part of PESSTO (the Public ESO Spectroscopic Survey for Transient Objects Survey) ESO program 188.D-3003, 191.D-0935, 197.D-1075. We thank the Las Cumbres Observatory and its staff for its continuing support of the ASAS-SN project. ASAS-SN is supported by the Gordon and Betty Moore Foundation through grant GBMF5490 to the Ohio State University and NSF grant AST-1515927. Development of ASAS-SN has been supported by NSF grant AST-0908816, the Mt. Cuba Astronomical Foundation, the Center for Cosmology and AstroParticle Physics at the Ohio State University, the Chinese Academy of Sciences South America Center for Astronomy (CAS- SACA), the Villum Foundation, and George Skestos. This paper uses data products produced by the OIR Telescope Data Center, supported by the Smithsonian Astrophysical Observatory. UKIRT is owned by the University of Hawaii (UH) and operated by the UH Institute for Astronomy; operations are enabled through the cooperation of the East Asian Observatory. When (some of) the data reported here were acquired, UKIRT was supported by NASA and operated under an agreement among the University of Hawaii, the University of Arizona, and Lockheed Martin Advanced Technology Center; operations were enabled through the cooperation of the East Asian Observatory.

Software: Python, Astropy (Astropy Collaboration et al. 2013), Matplotlib (Barrett et al. 2005), ISIS image subtraction package (Alard \& Lupton 1998; Alard 2000), DoPHOT (Schechter et al. 1993), HEASOFT (Nasa High Energy Astrophysics Science Archive Research Center (Heasarc), 2014), IRAF (Tody 1986, 1993).

\section{Appendix A Imaging Observations and Photometry}

ASAS-SN images are processed with an automated pipeline using the ISIS image subtraction package (Alard \& Lupton 1998; 
Table 4

LCOGT BVri Magnitudes of ASASSN-15pz

\begin{tabular}{|c|c|c|c|c|c|c|}
\hline Date & JD & Phase $^{\mathrm{a}}$ (day) & $B$ (mag) & $V$ (mag) & SDSS- $r$ (mag) & $\overline{\mathrm{SDSS}}-i$ (mag) \\
\hline 2015 Sep 28 & 2457294.2712 & -12.95 & $15.61 \pm 0.09$ & $15.49 \pm 0.05$ & $15.59 \pm 0.05$ & $15.77 \pm 0.07$ \\
\hline 2015 Sep 30 & 2457295.6212 & -11.60 & $15.21 \pm 0.04$ & $15.15 \pm 0.03$ & $15.26 \pm 0.04$ & $15.54 \pm 0.03$ \\
\hline 2015 Oct 1 & 2457297.0102 & -10.21 & $14.99 \pm 0.03$ & $14.91 \pm 0.04$ & $15.04 \pm 0.03$ & $15.32 \pm 0.05$ \\
\hline 2015 Oct 2 & 2457298.4970 & -8.72 & $14.73 \pm 0.05$ & $14.66 \pm 0.07$ & $14.87 \pm 0.10$ & $15.12 \pm 0.08$ \\
\hline 2015 Oct 4 & 2457300.3482 & -6.87 & $14.52 \pm 0.03$ & $14.51 \pm 0.02$ & $14.64 \pm 0.04$ & $\ldots$ \\
\hline 2015 Oct 6 & 2457302.3425 & -4.88 & $14.36 \pm 0.02$ & $14.36 \pm 0.02$ & $14.51 \pm 0.05$ & $14.95 \pm 0.03$ \\
\hline 2015 Oct 8 & 2457304.3372 & -2.88 & $14.28 \pm 0.02$ & $14.28 \pm 0.03$ & $14.41 \pm 0.06$ & $14.87 \pm 0.04$ \\
\hline 2015 Oct 10 & 2457306.3317 & -0.89 & $14.23 \pm 0.02$ & $14.23 \pm 0.02$ & $14.37 \pm 0.03$ & $14.86 \pm 0.03$ \\
\hline 2015 Oct 12 & 2457308.3824 & 1.16 & $14.19 \pm 0.05$ & $14.23 \pm 0.03$ & $14.36 \pm 0.03$ & $14.87 \pm 0.04$ \\
\hline 2015 Oct 15 & 2457310.9940 & 3.77 & $14.32 \pm 0.04$ & $14.28 \pm 0.02$ & $14.38 \pm 0.03$ & $14.87 \pm 0.05$ \\
\hline 2015 Oct 17 & 2457313.1149 & 5.89 & $14.34 \pm 0.04$ & $14.34 \pm 0.08$ & $14.41 \pm 0.03$ & $14.91 \pm 0.07$ \\
\hline 2015 Oct 20 & 2457316.3547 & 9.13 & $14.50 \pm 0.02$ & $14.40 \pm 0.03$ & $\ldots$ & $14.95 \pm 0.03$ \\
\hline 2015 Oct 24 & 2457319.5479 & 12.33 & $14.68 \pm 0.03$ & $14.51 \pm 0.03$ & $14.60 \pm 0.04$ & $15.04 \pm 0.05$ \\
\hline 2015 Oct 26 & 2457322.3168 & 15.10 & $14.91 \pm 0.03$ & $\ldots$ & $14.72 \pm 0.04$ & $15.16 \pm 0.04$ \\
\hline 2015 Oct 29 & 2457325.2811 & 18.06 & $15.12 \pm 0.04$ & $14.73 \pm 0.03$ & $14.79 \pm 0.04$ & $15.16 \pm 0.04$ \\
\hline 2015 Nov 1 & 2457327.7639 & 20.54 & $15.30 \pm 0.03$ & $14.84 \pm 0.05$ & $14.87 \pm 0.04$ & $15.15 \pm 0.04$ \\
\hline 2015 Nov 3 & 2457330.2885 & 23.07 & $15.47 \pm 0.01$ & $14.93 \pm 0.03$ & $14.89 \pm 0.03$ & $15.13 \pm 0.04$ \\
\hline 2015 Nov 6 & 2457333.0766 & 25.86 & $15.69 \pm 0.05$ & $15.05 \pm 0.04$ & $14.96 \pm 0.03$ & $15.16 \pm 0.04$ \\
\hline 2015 Nov 9 & 2457336.0004 & 28.78 & $15.92 \pm 0.06$ & $15.17 \pm 0.04$ & $15.06 \pm 0.09$ & $15.13 \pm 0.08$ \\
\hline 2015 Nov 15 & 2457342.1386 & 34.92 & $16.36 \pm 0.04$ & $15.52 \pm 0.03$ & $15.31 \pm 0.04$ & $15.32 \pm 0.04$ \\
\hline 2015 Nov 18 & 2457345.0433 & 37.82 & $\ldots$ & $15.66 \pm 0.03$ & $15.45 \pm 0.04$ & $15.45 \pm 0.05$ \\
\hline 2015 Nov 19 & 2457345.5801 & 38.36 & $16.61 \pm 0.05$ & $15.68 \pm 0.03$ & $15.45 \pm 0.03$ & $15.47 \pm 0.04$ \\
\hline 2015 Nov 21 & 2457348.0275 & 40.81 & $\ldots$ & $15.79 \pm 0.03$ & $15.57 \pm 0.04$ & $15.62 \pm 0.04$ \\
\hline 2015 Nov 24 & 2457351.0198 & 43.80 & $16.74 \pm 0.06$ & $15.89 \pm 0.04$ & $15.73 \pm 0.04$ & $15.77 \pm 0.05$ \\
\hline 2015 Nov 27 & 2457354.0115 & 46.79 & $\ldots$ & $\ldots$ & $15.84 \pm 0.06$ & $\ldots$ \\
\hline 2015 Nov 29 & 2457356.0039 & 48.78 & $16.91 \pm 0.04$ & $16.00 \pm 0.04$ & $15.87 \pm 0.03$ & $15.93 \pm 0.04$ \\
\hline 2015 Dec 1 & 2457358.4187 & 51.20 & $16.92 \pm 0.06$ & $16.09 \pm 0.04$ & $15.97 \pm 0.04$ & $16.03 \pm 0.05$ \\
\hline 2015 Dec 4 & 2457361.2791 & 54.06 & $17.01 \pm 0.04$ & $16.18 \pm 0.03$ & $16.08 \pm 0.03$ & $16.23 \pm 0.05$ \\
\hline 2015 Dec 6 & 2457363.2821 & 56.06 & $17.05 \pm 0.05$ & $16.22 \pm 0.05$ & $16.12 \pm 0.04$ & $16.23 \pm 0.05$ \\
\hline 2015 Dec 8 & 2457365.3853 & 58.17 & $17.15 \pm 0.05$ & $16.28 \pm 0.04$ & $16.18 \pm 0.04$ & $16.32 \pm 0.06$ \\
\hline 2015 Dec 11 & 2457368.3814 & 61.16 & $17.20 \pm 0.05$ & $16.36 \pm 0.03$ & $16.32 \pm 0.04$ & $16.44 \pm 0.04$ \\
\hline 2015 Dec 16 & 2457372.6042 & 65.38 & $17.24 \pm 0.07$ & $16.44 \pm 0.05$ & $16.44 \pm 0.04$ & $16.57 \pm 0.06$ \\
\hline 2015 Dec 18 & 2457375.0004 & 67.78 & $17.31 \pm 0.10$ & $16.53 \pm 0.07$ & $16.43 \pm 0.09$ & $16.62 \pm 0.07$ \\
\hline 2015 Dec 21 & 2457378.2918 & 71.07 & $17.38 \pm 0.11$ & $16.68 \pm 0.07$ & $16.53 \pm 0.09$ & $16.87 \pm 0.10$ \\
\hline 2015 Dec 24 & 2457380.9518 & 73.73 & $17.52 \pm 0.10$ & $16.70 \pm 0.07$ & $16.68 \pm 0.06$ & $\ldots$ \\
\hline 2015 Dec 26 & 2457383.3157 & 76.10 & $17.55 \pm 0.06$ & $16.73 \pm 0.04$ & $16.77 \pm 0.04$ & $16.98 \pm 0.07$ \\
\hline 2015 Dec 29 & 2457386.1151 & 78.90 & $17.56 \pm 0.08$ & $\ldots$ & $\ldots$ & $\ldots$ \\
\hline 2015 Dec 30 & 2457387.1286 & 79.91 & $\ldots$ & $\ldots$ & $\ldots$ & $17.07 \pm 0.10$ \\
\hline 2016 Jan 1 & 2457389.3087 & 82.09 & $17.64 \pm 0.06$ & $\ldots$ & $\ldots$ & $17.22 \pm 0.07$ \\
\hline 2016 Jan 2 & 2457390.3237 & 83.10 & $17.68 \pm 0.08$ & $16.97 \pm 0.04$ & $17.00 \pm 0.04$ & $17.25 \pm 0.07$ \\
\hline 2016 Jan 4 & 2457392.4078 & 85.19 & $17.71 \pm 0.10$ & $17.02 \pm 0.07$ & & $17.24 \pm 0.09$ \\
\hline 2016 Jan 7 & 2457395.3091 & 88.09 & $17.77 \pm 0.07$ & $17.04 \pm 0.05$ & $17.13 \pm 0.07$ & $17.28 \pm 0.10$ \\
\hline 2016 Jan 9 & 2457396.6167 & 89.40 & $17.77 \pm 0.07$ & $17.11 \pm 0.06$ & $17.19 \pm 0.09$ & $17.40 \pm 0.08$ \\
\hline 2016 Jan 10 & 2457398.3758 & 91.16 & $17.88 \pm 0.06$ & $17.18 \pm 0.04$ & $17.30 \pm 0.05$ & $17.56 \pm 0.10$ \\
\hline 2016 Jan 17 & 2457404.6003 & 97.38 & $18.01 \pm 0.09$ & $17.37 \pm 0.06$ & $17.53 \pm 0.08$ & $17.56 \pm 0.09$ \\
\hline 2016 Jan 23 & 2457411.0425 & 103.82 & $18.11 \pm 0.14$ & $17.56 \pm 0.08$ & $17.67 \pm 0.08$ & $17.91 \pm 0.14$ \\
\hline 2016 Jan 27 & 2457415.2879 & 108.07 & $18.20 \pm 0.07$ & $17.54 \pm 0.06$ & $17.88 \pm 0.05$ & $18.01 \pm 0.09$ \\
\hline 2016 Feb 6 & 2457425.3006 & 118.08 & $18.38 \pm 0.16$ & $\ldots$ & $18.27 \pm 0.10$ & $18.20 \pm 0.23$ \\
\hline 2016 Feb 11 & 2457430.3590 & 123.14 & $18.49 \pm 0.03$ & $18.01 \pm 0.09$ & $18.42 \pm 0.09$ & $18.39 \pm 0.16$ \\
\hline 2016 Feb 18 & 2457437.3258 & 130.11 & $18.63 \pm 0.06$ & $18.10 \pm 0.04$ & $18.47 \pm 0.06$ & $18.63 \pm 0.08$ \\
\hline 2016 Feb 22 & 2457441.2663 & 134.05 & $18.74 \pm 0.12$ & $18.18 \pm 0.06$ & $18.80 \pm 0.15$ & $18.68 \pm 0.10$ \\
\hline 2016 Mar 1 & 2457449.2720 & 142.05 & $18.86 \pm 0.06$ & $18.35 \pm 0.06$ & $18.91 \pm 0.06$ & $18.94 \pm 0.10$ \\
\hline 2016 Mar 6 & 2457453.5411 & 146.32 & $\ldots$ & $18.53 \pm 0.06$ & $\ldots$ & $19.25 \pm 0.16$ \\
\hline 2016 Mar 12 & 2457460.2677 & 153.05 & $19.04 \pm 0.08$ & $18.67 \pm 0.07$ & $19.44 \pm 0.11$ & $19.21 \pm 0.15$ \\
\hline 2016 Mar 17 & 2457465.2448 & 158.02 & $19.11 \pm 0.08$ & $18.72 \pm 0.07$ & $19.43 \pm 0.15$ & $19.40 \pm 0.15$ \\
\hline 2016 Mar 22 & 2457470.2461 & 163.03 & $\ldots$ & $18.95 \pm 0.15$ & $\ldots$ & $19.57 \pm 0.29$ \\
\hline
\end{tabular}

Notes.

${ }^{\text {a }}$ Relative to $B$-band peak time at JD $=2457307.2$.

Alard 2000) and calibrated with the AAVSO Photometric All-sky Survey (APASS; Henden et al. 2015). Optical images of ASASSN-15pz are primarily from the Las Cumbres Observatory Global Telescope Network (LCOGT; Brown et al. 2013) $1 \mathrm{~m}$ telescopes at the Siding Spring Observatory (SSO), South African Astronomical Observatory (SAAO) and Cerro Tololo Interamerican Observatory (CTIO). We also obtained optical and simultaneous near-infrared (NIR) data with A Novel Dual 
Table 5

SMARTS, NOT and UKIRT Magnitudes of ASASSN-15pz

\begin{tabular}{|c|c|c|c|c|c|c|c|c|}
\hline Date & JD & Phase $^{a}$ (day) & $B$ (mag) & $V$ (mag) & $J$ (mag) & $H$ (mag) & $K(\mathrm{mag})$ & Instrument \\
\hline 2015 Oct 23 & 2457318.7216 & 11.50 & $14.74 \pm 0.07$ & $14.50 \pm 0.07$ & $15.11 \pm 0.05$ & $14.87 \pm 0.05$ & $\cdots$ & ANDICAM $^{\mathrm{b}}$ \\
\hline 2015 Oct 27 & 2457322.6689 & 15.45 & $15.02 \pm 0.07$ & $14.63 \pm 0.07$ & $15.39 \pm 0.05$ & $14.72 \pm 0.05$ & $\cdots$ & ANDICAM \\
\hline 2015 Oct 28 & 2457323.5641 & 16.34 & $\ldots$ & $\ldots$ & $15.28 \pm 0.06$ & $14.79 \pm 0.12$ & $14.67 \pm 0.09$ & NOTCam ${ }^{\mathrm{c}}$ \\
\hline 2015 Oct 30 & 2457325.9293 & 18.71 & $\ldots$ & $\ldots$ & $15.36 \pm 0.11$ & $14.73 \pm 0.15$ & $14.78 \pm 0.20$ & $\mathrm{UFTI}^{\mathrm{d}}$ \\
\hline 2015 Nov 1 & 2457327.6091 & 20.39 & $15.40 \pm 0.07$ & $14.83 \pm 0.07$ & $15.51 \pm 0.06$ & $14.79 \pm 0.06$ & $\ldots$ & ANDICAM \\
\hline 2015 Nov 3 & 2457329.6998 & 22.48 & $15.58 \pm 0.06$ & $14.93 \pm 0.06$ & $15.44 \pm 0.05$ & $14.79 \pm 0.05$ & $\cdots$ & ANDICAM \\
\hline 2015 Nov 6 & 2457332.6043 & 25.38 & $15.79 \pm 0.07$ & $15.04 \pm 0.07$ & $15.51 \pm 0.06$ & $14.74 \pm 0.05$ & $\ldots$ & ANDICAM \\
\hline 2015 Nov 8 & 2457334.7238 & 27.50 & $15.95 \pm 0.07$ & $15.13 \pm 0.07$ & $15.51 \pm 0.05$ & $14.72 \pm 0.05$ & $\cdots$ & ANDICAM \\
\hline 2015 Nov 8 & 2457334.9057 & 27.69 & $\ldots$ & $\ldots$ & $15.40 \pm 0.10$ & $14.55 \pm 0.15$ & $14.84 \pm 0.20$ & UFTI \\
\hline 2015 Nov 10 & 2457336.6209 & 29.40 & $16.09 \pm 0.06$ & $15.24 \pm 0.06$ & $\ldots$ & $14.70 \pm 0.06$ & $\ldots$ & ANDICAM \\
\hline 2015 Nov 10 & 2457336.9105 & 29.69 & $\ldots$ & $\ldots$ & $15.39 \pm 0.10$ & $14.62 \pm 0.15$ & $14.87 \pm 0.20$ & UFTI \\
\hline 2015 Nov 14 & 2457340.7524 & 33.53 & $16.45 \pm 0.07$ & $15.46 \pm 0.07$ & $15.47 \pm 0.05$ & $14.71 \pm 0.06$ & $\cdots$ & ANDICAM \\
\hline 2015 Nov 16 & 2457342.6969 & 35.48 & $16.61 \pm 0.07$ & $15.59 \pm 0.07$ & $\ldots$ & $\cdots$ & $\cdots$ & ANDICAM \\
\hline 2015 Nov 20 & 2457346.7306 & 39.51 & $16.76 \pm 0.07$ & $15.76 \pm 0.07$ & $\ldots$ & $\cdots$ & $\cdots$ & ANDICAM \\
\hline 2015 Nov 21 & 2457347.5969 & 40.38 & $16.81 \pm 0.07$ & $15.78 \pm 0.07$ & $15.57 \pm 0.06$ & $\cdots$ & $\cdots$ & ANDICAM \\
\hline 2015 Nov 23 & 2457349.7622 & 42.54 & $16.86 \pm 0.07$ & $15.85 \pm 0.07$ & $15.77 \pm 0.06$ & $14.89 \pm 0.06$ & & ANDICAM \\
\hline 2015 Nov 24 & 2457350.5149 & 43.29 & $\ldots$ & $\ldots$ & $15.81 \pm 0.07$ & $15.07 \pm 0.10$ & $15.07 \pm 0.15$ & NOTCam \\
\hline 2015 Nov 27 & 2457353.7222 & 46.50 & $16.98 \pm 0.07$ & $15.98 \pm 0.07$ & $\cdots$ & $\cdots$ & $\cdots$ & ANDICAM \\
\hline 2015 Nov 29 & 2457355.6936 & 48.47 & $17.02 \pm 0.07$ & $16.03 \pm 0.07$ & $16.14 \pm 0.06$ & $\cdots$ & $\cdots$ & ANDICAM \\
\hline 2015 Nov 30 & 2457356.6619 & 49.44 & $17.03 \pm 0.06$ & $16.06 \pm 0.06$ & $16.17 \pm 0.06$ & $15.10 \pm 0.06$ & $\cdots$ & ANDICAM \\
\hline 2015 Dec 3 & 2457359.7406 & 52.52 & $17.07 \pm 0.08$ & $16.14 \pm 0.08$ & $\ldots$ & $\ldots$ & $\cdots$ & ANDICAM \\
\hline 2015 Dec 5 & 2457361.6426 & 54.42 & $17.13 \pm 0.06$ & $16.20 \pm 0.06$ & $16.37 \pm 0.06$ & $15.31 \pm 0.06$ & $\cdots$ & ANDICAM \\
\hline 2015 Dec 6 & 2457362.6341 & 55.41 & $17.13 \pm 0.06$ & $16.21 \pm 0.06$ & $16.46 \pm 0.06$ & $15.38 \pm 0.06$ & $\cdots$ & ANDICAM \\
\hline 2015 Dec 8 & 2457364.6270 & 57.41 & $17.17 \pm 0.07$ & $16.27 \pm 0.07$ & $16.62 \pm 0.07$ & $15.38 \pm 0.06$ & $\cdots$ & ANDICAM \\
\hline 2015 Dec 11 & 2457367.6116 & 60.39 & $17.26 \pm 0.07$ & $16.35 \pm 0.07$ & $16.68 \pm 0.07$ & $\cdots$ & $\cdots$ & ANDICAM \\
\hline 2015 Dec 15 & 2457371.6651 & 64.45 & $17.31 \pm 0.07$ & $16.45 \pm 0.07$ & $16.90 \pm 0.07$ & $15.54 \pm 0.07$ & $\cdots$ & ANDICAM \\
\hline 2015 Dec 17 & 2457373.5966 & 66.38 & $17.36 \pm 0.07$ & $16.52 \pm 0.07$ & $17.16 \pm 0.09$ & $15.61 \pm 0.07$ & $\cdots$ & ANDICAM \\
\hline 2015 Dec 21 & 2457377.5802 & 70.36 & $17.45 \pm 0.07$ & $16.61 \pm 0.07$ & $17.24 \pm 0.09$ & $15.82 \pm 0.07$ & $\cdots$ & ANDICAM \\
\hline 2015 Dec 22 & 2457379.4459 & 72.23 & $\ldots$ & $\ldots$ & $\ldots$ & $\ldots$ & $15.36 \pm 0.17$ & NOTCam \\
\hline 2015 Dec 23 & 2457379.5940 & 72.37 & $17.47 \pm 0.07$ & $16.65 \pm 0.07$ & $\cdots$ & $\cdots$ & $\cdots$ & ANDICAM \\
\hline 2015 Dec 24 & 2457380.7604 & 73.54 & $\ldots$ & $\ldots$ & $17.25 \pm 0.05$ & $15.90 \pm 0.08$ & $15.35 \pm 0.07$ & WFCAM $^{\mathrm{e}}$ \\
\hline 2015 Dec 28 & 2457384.6249 & 77.40 & $17.59 \pm 0.07$ & $16.78 \pm 0.07$ & $17.57 \pm 0.11$ & $\ldots$ & $\ldots$ & ANDICAM \\
\hline 2015 Dec 30 & 2457386.6397 & 79.42 & $17.63 \pm 0.07$ & $16.84 \pm 0.07$ & $\cdots$ & $\cdots$ & $\cdots$ & ANDICAM \\
\hline 2016 Jan 1 & 2457388.5718 & 81.35 & $17.67 \pm 0.07$ & $16.87 \pm 0.07$ & $\cdots$ & $\cdots$ & $\cdots$ & ANDICAM \\
\hline 2016 Jan 1 & 2457388.7285 & 81.51 & $\ldots$ & $\ldots$ & $17.59 \pm 0.05$ & $16.14 \pm 0.08$ & $15.46 \pm 0.07$ & WFCAM \\
\hline 2016 Jan 3 & 2457390.5613 & 83.34 & $17.67 \pm 0.07$ & $16.92 \pm 0.07$ & $\ldots$ & $\ldots$ & $\ldots$ & ANDICAM \\
\hline 2016 Jan 3 & 2457390.7389 & 83.52 & $\ldots$ & $\ldots$ & $17.67 \pm 0.05$ & $16.22 \pm 0.08$ & $15.50 \pm 0.07$ & WFCAM \\
\hline 2016 Jan 7 & 2457394.6230 & 87.40 & $17.80 \pm 0.07$ & $17.07 \pm 0.07$ & $\ldots$ & $\ldots$ & $\ldots$ & ANDICAM \\
\hline 2016 Jan 9 & 2457396.5623 & 89.34 & $17.81 \pm 0.07$ & $17.09 \pm 0.07$ & $\cdots$ & $\cdots$ & $\cdots$ & ANDICAM \\
\hline 2016 Jan 11 & 2457398.5604 & 91.34 & $17.88 \pm 0.07$ & $17.15 \pm 0.07$ & $\cdots$ & $\cdots$ & $\cdots$ & ANDICAM \\
\hline 2016 Jan 13 & 2457400.5831 & 93.36 & $17.92 \pm 0.07$ & $17.21 \pm 0.07$ & $\cdots$ & $\cdots$ & $\cdots$ & ANDICAM \\
\hline 2016 Jan 15 & 2457402.5868 & 95.37 & $17.98 \pm 0.07$ & $17.25 \pm 0.07$ & $\cdots$ & $\cdots$ & $\cdots$ & ANDICAM \\
\hline 2016 Jan 17 & 2457404.5331 & 97.31 & $18.02 \pm 0.07$ & $17.32 \pm 0.07$ & $\cdots$ & $\cdots$ & $\cdots$ & ANDICAM \\
\hline 2016 Jan 18 & 2457405.7279 & 98.51 & $\ldots$ & $\ldots$ & $18.39 \pm 0.06$ & $16.77 \pm 0.08$ & $15.88 \pm 0.09$ & WFCAM \\
\hline 2016 Jan 19 & 2457406.5906 & 99.37 & $18.05 \pm 0.07$ & $99.90 \pm 0.07$ & $\cdots$ & $\cdots$ & $\cdots$ & ANDICAM \\
\hline 2016 Jan 21 & 2457408.5669 & 101.35 & $18.10 \pm 0.06$ & $17.42 \pm 0.06$ & $\cdots$ & $\ldots$ & $\cdots$ & ANDICAM \\
\hline 2016 Jan 24 & 2457412.3189 & 105.10 & $\cdots$ & $\ldots$ & $18.57 \pm 0.08$ & $16.92 \pm 0.15$ & $\cdots$ & NOTCam \\
\hline 2016 Jan 26 & 2457413.6057 & 106.39 & $18.22 \pm 0.10$ & $17.56 \pm 0.10$ & $\ldots$ & $\ldots$ & $\cdots$ & ANDICAM \\
\hline
\end{tabular}

Notes.

a Relative to $B$-band peak time at JD $=2457307.2$.

b Optical and NIR camera on the SMARTS $1.3 \mathrm{~m}$ telescope.

${ }^{\mathrm{c}}$ Near-infrared Camera and spectrograph on the Nordic Optical Telescope (NOT; $2.56 \mathrm{~m}$ ).

d NIR imaging camera on the United Kingdom Infrared Telescope (UKIRT; $3.8 \mathrm{~m}$ ).

e NIR imaging camera on the United Kingdom Infrared Telescope (UKIRT; $3.8 \mathrm{~m}$ ).

Imaging CAMera (ANDICAM; DePoy et al. 2003) on the $1.3 \mathrm{~m}$ Small \& Moderate Aperture Research Telescope System (SMARTS; Subasavage et al. 2010) at Cerro Tololo, Chile. In addition, we obtained NIR imaging observations with the NOTCam on Nordic Optical Telescope (NOT), the UKIRT Fast Track Imager (UFTI) and the Wide Field CAMera (WFCAM;
Casali et al. 2007) on the United Kingdom Infrared Telescope (UKIRT). The optical and near-infrared images were reduced after bias/dark-frame and flat-field corrections. We use DoPHOT (Schechter et al. 1993) to perform Point-spread-function (PSF) photometry, which is sufficient for ASASSN-15pz given the very low host-galaxy background. For the optical bands (Johnson $B$, 
Table 6

Swift UVOT Photometry of ASASSN-15pz

\begin{tabular}{|c|c|c|c|c|c|c|c|}
\hline Date & JD & uvw2 & uvm2 & $u v w 1$ & $u$ & $b$ & $v$ \\
\hline 2015 Oct 14 & 2457309.8480 & $15.75 \pm 0.05$ & $15.60 \pm 0.04$ & $14.60 \pm 0.04$ & $13.45 \pm 0.04$ & $14.24 \pm 0.03$ & $14.24 \pm 0.04$ \\
\hline 2015 Oct 17 & 2457312.5954 & $15.95 \pm 0.05$ & $15.94 \pm 0.05$ & $14.89 \pm 0.04$ & $13.66 \pm 0.04$ & $14.30 \pm 0.03$ & $14.35 \pm 0.04$ \\
\hline 2015 Oct 20 & 2457315.7142 & $16.36 \pm 0.05$ & $16.39 \pm 0.05$ & $15.25 \pm 0.05$ & $13.97 \pm 0.04$ & $14.46 \pm 0.03$ & $14.41 \pm 0.04$ \\
\hline 2015 Oct 23 & 2457318.7697 & $16.85 \pm 0.06$ & $16.85 \pm 0.06$ & $15.65 \pm 0.05$ & $14.37 \pm 0.04$ & $14.65 \pm 0.03$ & $14.50 \pm 0.04$ \\
\hline 2015 Oct 26 & 2457321.7614 & $17.09 \pm 0.07$ & $17.05 \pm 0.06$ & $15.96 \pm 0.05$ & $14.69 \pm 0.04$ & $14.84 \pm 0.03$ & $14.68 \pm 0.04$ \\
\hline 2015 Oct 29 & 2457324.6257 & $17.15 \pm 0.07$ & $17.16 \pm 0.07$ & $16.32 \pm 0.06$ & $14.99 \pm 0.04$ & $15.01 \pm 0.04$ & $14.68 \pm 0.04$ \\
\hline 2015 Nov 1 & 2457327.6927 & $17.51 \pm 0.12$ & $\ldots$ & $16.50 \pm 0.07$ & $15.30 \pm 0.05$ & $15.27 \pm 0.04$ & $\ldots$ \\
\hline 2015 Nov 4 & 2457330.5998 & $17.98 \pm 0.09$ & $17.66 \pm 0.08$ & $16.70 \pm 0.07$ & $15.56 \pm 0.05$ & $15.50 \pm 0.04$ & $14.94 \pm 0.04$ \\
\hline 2015 Nov 7 & 2457333.7948 & $17.89 \pm 0.09$ & $17.55 \pm 0.08$ & $16.86 \pm 0.07$ & $15.81 \pm 0.05$ & $15.74 \pm 0.04$ & $15.09 \pm 0.04$ \\
\hline 2015 Nov 17 & 2457344.3791 & $18.23 \pm 0.12$ & $17.86 \pm 0.10$ & $17.48 \pm 0.10$ & $16.60 \pm 0.08$ & $16.54 \pm 0.06$ & $15.60 \pm 0.06$ \\
\hline 2015 Nov 22 & 2457349.2347 & $18.39 \pm 0.15$ & $17.86 \pm 0.11$ & $17.75 \pm 0.12$ & $16.90 \pm 0.10$ & $16.66 \pm 0.07$ & $15.85 \pm 0.07$ \\
\hline 2015 Nov 27 & 2457354.2926 & $\ldots$ & $\ldots$ & $17.83 \pm 0.12$ & $17.02 \pm 0.10$ & $\ldots$ & $\ldots$ \\
\hline 2015 Dec 2 & 2457359.0546 & $18.72 \pm 0.20$ & $18.51 \pm 0.17$ & $17.88 \pm 0.15$ & $17.12 \pm 0.12$ & $16.93 \pm 0.08$ & $16.19 \pm 0.09$ \\
\hline 2015 Dec 7 & 2457363.7879 & $18.88 \pm 0.17$ & $18.46 \pm 0.13$ & $17.94 \pm 0.12$ & $17.29 \pm 0.10$ & $17.05 \pm 0.07$ & $16.22 \pm 0.07$ \\
\hline 2015 Dec 12 & 2457369.3009 & $19.16 \pm 0.22$ & $18.92 \pm 0.19$ & $17.94 \pm 0.13$ & $17.48 \pm 0.12$ & $17.24 \pm 0.08$ & $16.45 \pm 0.09$ \\
\hline 2015 Dec 17 & 2457373.6811 & $18.82 \pm 0.20$ & $18.88 \pm 0.21$ & $18.02 \pm 0.17$ & $17.53 \pm 0.15$ & $17.27 \pm 0.09$ & $16.66 \pm 0.12$ \\
\hline 2015 Dec 22 & 2457379.1334 & $19.21 \pm 0.20$ & $18.79 \pm 0.14$ & $18.19 \pm 0.14$ & $17.53 \pm 0.11$ & $17.41 \pm 0.07$ & $16.67 \pm 0.09$ \\
\hline 2015 Dec 27 & 2457383.9241 & $19.17 \pm 0.20$ & $19.00 \pm 0.17$ & $18.43 \pm 0.16$ & $17.77 \pm 0.13$ & $17.50 \pm 0.08$ & $16.76 \pm 0.10$ \\
\hline 2016 Jan 1 & 2457388.8610 & $19.40 \pm 0.23$ & $19.08 \pm 0.19$ & $18.84 \pm 0.21$ & $17.93 \pm 0.15$ & $17.40 \pm 0.08$ & $17.12 \pm 0.12$ \\
\hline 2016 Jan 6 & 2457394.2360 & $19.36 \pm 0.22$ & $19.00 \pm 0.17$ & $18.83 \pm 0.20$ & $17.95 \pm 0.14$ & $17.73 \pm 0.09$ & $17.21 \pm 0.12$ \\
\hline 2016 Jan 10 & 2457398.2922 & $19.63 \pm 0.26$ & $19.65 \pm 0.26$ & $18.80 \pm 0.20$ & $18.47 \pm 0.22$ & $17.81 \pm 0.10$ & $17.22 \pm 0.12$ \\
\hline 2016 Jan 28 & 2457415.6343 & $\ldots$ & $19.63 \pm 0.25$ & $19.39 \pm 0.29$ & $18.84 \pm 0.26$ & $18.02 \pm 0.11$ & $17.68 \pm 0.16$ \\
\hline 2016 Feb 7 & 2457425.6012 & $\cdots$ & $\cdots$ & $\ldots$ & $18.81 \pm 0.45$ & $18.32 \pm 0.23$ & $17.63 \pm 0.24$ \\
\hline 2016 Feb 17 & 2457435.5204 & $\ldots$ & $\ldots$ & $\ldots$ & $19.00 \pm 0.32$ & $18.83 \pm 0.20$ & $17.95 \pm 0.21$ \\
\hline 2016 Feb 27 & 2457446.3459 & $\cdots$ & $\cdots$ & $\cdots$ & $19.66 \pm 0.57$ & $18.83 \pm 0.23$ & $18.02 \pm 0.24$ \\
\hline
\end{tabular}

$V$, and SDSS- $r, i$ ) the photometry data were calibrated using APASS, and the NIR photometry was calibrated using 2MASS (Skrutskie et al. 2006). We estimate the photometric uncertainties by adding the DoPHOT and the zero-point calibration uncertainties in quadrature. The optical and NIR photometric results are given in Tables 4 and 5, where the SDSS- $r$ and $i$ magnitudes are in the $\mathrm{AB}$ magnitude system and all the others are in the Vega system.

Target of Opportunity observations of ASASSN-15pz (target ID 34102) were performed between Oct 14th 2015 and Feb 27th 2016 with Swift/UVOT(Poole et al. 2008) in the $u v w 2$

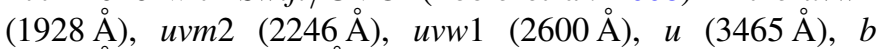
$(4392 \AA)$, and $v(5468 \AA$ ) filters. Host-galaxy template images were obtained in March 2017 (PI M. Stritzinger, target ID 87296) to estimate and then we subtracted the background galaxy contribution. We extracted source counts from a 5!"0 radius region centered on ASASSN-15pz using the UVOT software task uvotsource. The source counts were converted into the Vega magnitude system based on the most recent UVOT calibrations (Breeveld et al. 2011), and the UVOT magnitudes are listed in Table 6.

\section{Appendix B \\ Spectroscopic Observations}

Spectroscopic observations were made at eight different epochs, ranging in phase from -11.6 days (JD 2457295.66) to +239.7 days (JD 2457546.90), as summarized in Table 7. We made use of multiple instruments, including the $2.56 \mathrm{~m}$ Nordic Optical Telescope (NOT) with ALFOSC, the $2.5 \mathrm{~m}$ du Pont telescope with WFCCD, the $3.58 \mathrm{~m}$ New Technology Telescope (NTT) with EFOSC, and the $6.5 \mathrm{~m}$ Magellan telescopes with IMACS and LDSS3C. The NTT spectra were obtained as part of the Public ESO Spectroscopic Survey for Transient Objects (PESSTO, Smartt et al. 2015) program and were reduced using a custom pipeline as described in Smartt et al. (2015). The nebular-phase spectra taken at 151 days and 240 days with the Magellan telescopes are part of the 100IAS program (Dong et al. 2018). Spectroscopic data were reduced within the IRAF environment following standard procedures. Appropriate spectrophotometric standard stars were used to compute nightly response functions, which were used to fluxcalibrate the science spectra. 
Table 7

Log of Spectroscopic Observation of ASASSN-15pz

\begin{tabular}{|c|c|c|c|c|}
\hline Date & JD & Phase (day) & Range $(\AA)$ & Instrument \\
\hline 2015 Sep 30 & 2457295.66 & -11.6 & $3500-9000$ & $\mathrm{NOT}^{\mathrm{a}} /$ ALFOSC \\
\hline 2015 Nov 5 & 2457331.54 & +24.3 & $3500-9000$ & NOT/ALFOSC \\
\hline 2015 Nov 9 & 2457335.71 & +28.5 & $3700-9250$ & du Pont ${ }^{\mathrm{b}} /$ WFCCD \\
\hline 2015 Dec 17 & 2457373.74 & +66.5 & $3650-9250$ & $\mathrm{NTT}^{\mathrm{c}} / \mathrm{EFOSC}$ \\
\hline 2016 Jan 11 & 2457398.57 & +91.3 & $3700-9250$ & du Pont/WFCCD \\
\hline 2016 Feb 2 & 2457420.56 & +113.3 & $3700-9250$ & du Pont/WFCCD \\
\hline 2016 Feb 18 & 2457436.56 & +129.3 & $3650-9250$ & NTT/EFOSC \\
\hline 2016 Jun 7 & 2457546.90 & +239.7 & $4800-10000$ & $\mathrm{Clay}^{\mathrm{e}} / \mathrm{LDSS} 3 \mathrm{C}$ \\
\hline
\end{tabular}

Notes.

a Nordic Optical Telescope (2.56 m) located at Roque de los Muchachos Observatory, La Palma in the Canary Islands.

b du Pont telescope $(2.5 \mathrm{~m})$ located in Chile at Las Campanas Observatory.

${ }^{\mathrm{c}}$ New Technology Telescope $(3.58 \mathrm{~m})$ located in Chile at the La Silla Observatory.

d One of the Magellan Telescopes $(6.5 \mathrm{~m})$ located at Las Campanas Observatory in Chile.

e One of the Magellan Telescopes $(6.5 \mathrm{~m})$ located at Las Campanas Observatory in Chile.

\section{ORCID iDs}

Ping Chen (iD https://orcid.org/0000-0003-0853-6427

Subo Dong (iD https://orcid.org/0000-0002-1027-0990

Boaz Katz (iD https://orcid.org/0000-0003-0584-2920

C. S. Kochanek (i) https://orcid.org/0000-0001-6017-2961

Juna A. Kollmeier (iD https://orcid.org/0000-0001-9852-1610

M. M. Phillips (i) https://orcid.org/0000-0003-2734-0796

J. L. Prieto (i) https://orcid.org/0000-0003-0943-0026

B. J. Shappee (1) https://orcid.org/0000-0003-4631-1149

M. D. Stritzinger (1) https://orcid.org/0000-0002-5571-1833

Subhash Bose (iD https://orcid.org/0000-0003-3529-3854

Peter J. Brown (D) https://orcid.org/0000-0001-6272-5507

T. W.-S. Holoien (i) https://orcid.org/0000-0001-9206-3460

L. Galbany (1) https://orcid.org/0000-0002-1296-6887

Peter A. Milne (i) https://orcid.org/0000-0002-0370-157X

Nidia Morrell (i) https://orcid.org/0000-0003-2535-3091

Anthony L. Piro (i) https://orcid.org/0000-0001-6806-0673

D. R. Young (iD https://orcid.org/0000-0002-1229-2499

\section{References}

Alard, C. 2000, A\&AS, 144, 363

Alard, C., \& Lupton, R. H. 1998, ApJ, 503, 325

Arnett, W. D. 1982, ApJ, 253, 785

Astropy Collaboration, Robitaille, T. P., Tollerud, E. J., et al. 2013, A\&A, 558, A33

Axelrod, T. S. 1980, PhD thesis, California Univ., Santa Cruz

Barrett, P., Hunter, J., Miller, J. T., Hsu, J.-C., \& Greenfield, P. 2005, in ASP

Conf. Ser. 347, Astronomical Data Analysis Software and Systems XIV, ed.

P. Shopbell, M. Britton, \& R. Ebert (San Francisco, CA: ASP), 91

Benetti, S., Meikle, P., Stehle, M., et al. 2004, MNRAS, 348, 261

Blondin, S., Dessart, L., \& Hillier, D. J. 2018, MNRAS, 474, 3931

Blondin, S., Matheson, T., Kirshner, R. P., et al. 2012, AJ, 143, 126

Branch, D. 1992, ApJ, 392, 35

Branch, D., Chau Dang, L., \& Baron, E. 2009, PASP, 121, 238

Breeveld, A. A., Landsman, W., Holland, S. T., et al. 2011, in AIP Conf. Ser. 1358, Gamma Ray Bursts 2010, ed. J. E. McEnery, J. L. Racusin, \& N. Gehrels (Melville, NY: AIP), 373

Brimacombe, J., Marples, P., Kiyota, S., et al. 2015, ATel, 8099, 1

Brown, P. J., Breeveld, A. A., Holland, S., Kuin, P., \& Pritchard, T. 2014a, Ap\&SS, 354, 89

Brown, P. J., Holland, S. T., Immler, S., et al. 2009, AJ, 137, 4517

Brown, P. J., Kuin, P., Scalzo, R., et al. 2014b, ApJ, 787, 29

Brown, P. J., Roming, P. W. A., Milne, P., et al. 2010, ApJ, 721, 1608

Brown, T. M., Baliber, N., Bianco, F. B., et al. 2013, PASP, 125, 1031

Burns, C. R., Parent, E., Phillips, M. M., et al. 2018, ApJ, 869, 56
Burns, C. R., Stritzinger, M., Phillips, M. M., et al. 2014, ApJ, 789, 32 Casali, M., Adamson, A., Alves de Oliveira, C., et al. 2007, A\&A, 467, 777 Chakradhari, N. K., Sahu, D. K., Srivastav, S., \& Anupama, G. C. 2014, MNRAS, 443, 1663

Childress, M. J., Tucker, B. E., Yuan, F., et al. 2016, PASA, 33, e055 Contardo, G., Leibundgut, B., \& Vacca, W. D. 2000, A\&A, 359, 876

Contreras, C., Phillips, M. M., Burns, C. R., et al. 2018, ApJ, 859, 24 DePoy, D. L., Atwood, B., Belville, S. R., et al. 2003, Proc. SPIE, 4841, 827 Dong, S., Katz, B., Kollmeier, J. A., et al. 2018, MNRAS, 479, L70

Dong, S., Katz, B., Kushnir, D., \& Prieto, J. L. 2015, MNRAS, 454, L61

Filippenko, A. V., Richmond, M. W., Branch, D., et al. 1992a, AJ, 104, 1543

Filippenko, A. V., Richmond, M. W., Matheson, T., et al. 1992b, ApJL, 384, L15

Fink, M., Kromer, M., Hillebrandt, W., et al. 2018, A\&A, 618, A124

Folatelli, G., Phillips, M. M., Morrell, N., et al. 2012, ApJ, 745, 74

Friedman, A. S., Wood-Vasey, W. M., Marion, G. H., et al. 2015, ApJS, 220, 9

Gall, C., Stritzinger, M. D., Ashall, C., et al. 2018, A\&A, 611, A58

Gehrels, N., Chincarini, G., Giommi, P., et al. 2004, ApJ, 611, 1005

Gómez, G., \& López, R. 1998, AJ, 115, 1096

Hachinger, S., Mazzali, P. A., Taubenberger, S., et al. 2012, MNRAS, 427, 2057

Henden, A. A., Levine, S., Terrell, D., \& Welch, D. L. 2015, AAS Meeting Abstracts, 225, 336.16

Hicken, M., Garnavich, P. M., Prieto, J. L., et al. 2007, ApJL, 669, L17

Howell, D. A., Sullivan, M., Nugent, P. E., et al. 2006, Natur, 443, 308

Jeffery, D. J. 1999, arXiv:astro-ph/9907015

Jeffery, D. J., Leibundgut, B., Kirshner, R. P., et al. 1992, ApJ, 397, 304

Jha, S., Branch, D., Chornock, R., et al. 2006, AJ, 132, 189

Jones, D. H., Read, M. A., Saunders, W., et al. 2009, MNRAS, 399, 683

Katz, B., Kushnir, D., \& Dong, S. 2013, arXiv:1301.6766

Krisciunas, K., Contreras, C., Burns, C. R., et al. 2017, AJ, 154, 211

Kushnir, D., Katz, B., Dong, S., Livne, E., \& Fernández, R. 2013, ApJL, 778, L37

Leibundgut, B., Kirshner, R. P., Phillips, M. M., et al. 1993, AJ, 105, 301

Li, W., Filippenko, A. V., Chornock, R., et al. 2003, PASP, 115, 453

Maguire, K., Sim, S. A., Shingles, L., et al. 2018, MNRAS, 477, 3567

Maoz, D., Mannucci, F., \& Nelemans, G. 2014, ARA\&A, 52, 107

Mazzali, P. A. 2001, MNRAS, 321, 341

Mazzali, P. A., Cappellaro, E., Danziger, I. J., Turatto, M., \& Benetti, S. 1998, ApJL, 499, L49

Mazzali, P. A., Danziger, I. J., \& Turatto, M. 1995, A\&A, 297, 509

Mazzali, P. A., \& Hachinger, S. 2012, MNRAS, 424, 2926

Mazzali, P. A., Sullivan, M., Filippenko, A. V., et al. 2015, MNRAS, 450, 2631

Milne, P. A., Brown, P. J., Roming, P. W. A., Bufano, F., \& Gehrels, N. 2013, ApJ, 779, 23

Nasa High Energy Astrophysics Science Archive Research Center (Heasarc) 2014, HEAsoft: Unified Release of FTOOLS and XANADU, Astrophysics Source Code Library, ascl:1408.004

Noebauer, U. M., Taubenberger, S., Blinnikov, S., Sorokina, E., \& Hillebrandt, W. 2016, MNRAS, 463, 2972 
Nugent, P., Phillips, M., Baron, E., Branch, D., \& Hauschildt, P. 1995, ApJL, 455, L147

Parrent, J. T., Howell, D. A., Fesen, R. A., et al. 2016, MNRAS, 457, 3702

Parrent, J. T., Thomas, R. C., Fesen, R. A., et al. 2011, ApJ, 732, 30

Phillips, M. M. 1993, ApJL, 413, L105

Phillips, M. M. 2005, in ASP Conf. Ser. 342, 1604-2004: Supernovae as Cosmological Lighthouses, ed. M. Turatto et al. (San Francisco, CA: ASP), 211

Phillips, M. M. 2012, PASA, 29, 434

Phillips, M. M., Lira, P., Suntzeff, N. B., et al. 1999, AJ, 118, 1766

Phillips, M. M., Simon, J. D., Morrell, N., et al. 2013, ApJ, 779, 38

Poole, T. S., Breeveld, A. A., Page, M. J., et al. 2008, MNRAS, 383, 627

Poznanski, D., Prochaska, J. X., \& Bloom, J. S. 2012, MNRAS, 426, 1465

Pskovskii, I. P. 1977, SvA, 21, 675

Ruiz-Lapuente, P., Cappellaro, E., Turatto, M., et al. 1992, ApJL, 387, L33

Scalzo, R., Aldering, G., Antilogus, P., et al. 2012, ApJ, 757, 12

Scalzo, R. A., Aldering, G., Antilogus, P., et al. 2010, ApJ, 713, 1073

Schechter, P. L., Mateo, M., \& Saha, A. 1993, PASP, 105, 1342

Schlafly, E. F., \& Finkbeiner, D. P. 2011, ApJ, 737, 103

Schlegel, D. J., Finkbeiner, D. P., \& Davis, M. 1998, ApJ, 500, 525

Shappee, B. J., Prieto, J. L., Grupe, D., et al. 2014, ApJ, 788, 48

Silverman, J. M., Ganeshalingam, M., Li, W., et al. 2011, MNRAS, 410, 585

Skrutskie, M. F., Cutri, R. M., Stiening, R., et al. 2006, AJ, 131, 1163

Smartt, S. J., Valenti, S., Fraser, M., et al. 2015, A\&A, 579, A40
Stanishev, V., Goobar, A., Benetti, S., et al. 2007, A\&A, 469, 645

Stritzinger, M., \& Leibundgut, B. 2005, A\&A, 431, 423

Stritzinger, M., Leibundgut, B., Walch, S., \& Contardo, G. 2006, A\&A, 450,241

Subasavage, J. P., Bailyn, C. D., Smith, R. C., et al. 2010, Proc. SPIE, 7737, $77371 \mathrm{C}$

Taddia, F., \& Stritzinger, M. D. 2015, ATel, 8105, 1

Taubenberger, S. 2017, Handbook of Supernovae (Berlin: Springer)

Taubenberger, S., Benetti, S., Childress, M., et al. 2011, MNRAS, 412, 2735

Taubenberger, S., Kromer, M., Hachinger, S., et al. 2013, MNRAS, 432, 3117

Tody, D. 1986, Proc. SPIE, 627, 733

Tody, D. 1993, in ASP Conf. Ser. 52, Astronomical Data Analysis Software and Systems II, ed. R. J. Hanisch, R. J. V. Brissenden, \& J. Barnes (San Francisco, CA: ASP), 173

Turatto, M., Benetti, S., Cappellaro, E., et al. 1996, MNRAS, 283, 1

Wang, B. 2018, RAA, 18, 049

Wang, X., Li, W., Filippenko, A. V., et al. 2009, ApJ, 697, 380

Wygoda, N., Elbaz, Y., \& Katz, B. 2019a, MNRAS, 484, 3941

Wygoda, N., Elbaz, Y., \& Katz, B. 2019b, MNRAS, 484, 3951

Yamanaka, M., Kawabata, K. S., Kinugasa, K., et al. 2009a, ApJL, 707, L118

Yamanaka, M., Maeda, K., Tanaka, M., et al. 2016, PASJ, 68, 68

Yamanaka, M., Naito, H., Kinugasa, K., et al. 2009b, PASJ, 61, 713

Yaron, O., \& Gal-Yam, A. 2012, PASP, 124, 668 